\title{
KLF2 Protects against Osteoarthritis by Repressing Oxidative Response through Activation of Nrf2/ARE Signaling In Vitro and In Vivo
}

\author{
Xiang Gao $\mathbb{D}$, Shuangpeng Jiang $\mathbb{D}$, Zhangzhen Du $(\mathbb{D}$, Angtin $K e \mathbb{D}$, Qingwei Liang $\mathbb{D}$, \\ and $\mathrm{Xu} \mathrm{Li}$ \\ Department of Orthopedics, The First Hospital of China Medical University, Shenyang 110000, China \\ Correspondence should be addressed to Xu Li; likyokucmu@163.com
}

Received 13 August 2019; Accepted 17 October 2019; Published 19 November 2019

Academic Editor: Juan Gambini

Copyright (C) 2019 Xiang Gao et al. This is an open access article distributed under the Creative Commons Attribution License, which permits unrestricted use, distribution, and reproduction in any medium, provided the original work is properly cited.

\begin{abstract}
Osteoarthritis (OA) is a multifactorial and inflammatory disease characterized by cartilage destruction that can cause disability among aging patients. There is currently no effective treatment that can arrest or reverse OA progression. Kruppel-like factor 2 (KLF2), a member of the zinc finger family, has emerged as a transcription factor involved in a wide variety of inflammatory diseases. Here, we identified that KLF2 expression is downregulated in IL- $1 \beta$-treated human chondrocytes and OA cartilage. Genetic and pharmacological overexpression of KLF2 suppressed IL- $1 \beta$-induced apoptosis and matrix degradation through the suppression of reactive oxygen species (ROS) production. In addition, KLF2 overexpression resulted in increased expression of heme oxygenase-1 ( $\mathrm{HO}-1)$ and $\mathrm{NAD}(\mathrm{P}) \mathrm{H}$ dehydrogenase quinone 1 (NQO1) through the enhanced nuclear translocation of nuclear factor erythroid 2-related factor 2 (Nrf2). Further, Nrf2 inhibition abrogated the chondroprotective effects of KLF2. Safranin O/fast green and TUNEL staining demonstrated that adenovirus-mediated overexpression of KLF2 in joint cartilage protects rats against experimental OA by inhibiting cartilage degradation and chondrocyte apoptosis. Immunohistochemical staining revealed that KLF2 overexpression significantly decreases MMP13 expression caused by OA progression in vivo. This in vitro and in vivo study is the first to investigate the antioxidative effect and mechanisms of KLF2 in OA pathogenesis. Our results collectively provide new insights into OA pathogenesis regulated by KLF2 and a rationale for the development of effective OA intervention strategies.
\end{abstract}

\section{Introduction}

Osteoarthritis $(\mathrm{OA})$ is one of the most prevalent joint diseases and is predicted to be the greatest cause of disability among patients over 40 years old by 2040 [1]. Although the occurrence and development of OA have been studied extensively, there is currently no efficient treatment that can arrest or reverse OA progression. Hence, identifying an enhanced regulatory mechanism for cartilage homeostasis in OA will help in managing this disease using specific targets. OA is caused by a cartilage homeostasis disorder that can be induced by various physiological and mechanical factors, such as age, genetics, mechanical stress, trauma, and metabolism [2-5]. A hallmark of OA is the degradation of the extracellular matrix (ECM) [4], which is considered a direct outcome of the action of matrix-degrading enzymes (MMPs). Among the MMPs, MMP3, MMP9, and MMP13 are known to play crucial roles in OA cartilage destruction $[6,7]$. The apoptosis of chondrocytes (a unique resident cell type in articular cartilage) also greatly contributes to the degradation of the ECM [8]. Because the release of MMPs and apoptosis of chondrocytes are indispensable components of OA progression, it is reasonable to hypothesize that efficient attenuation of these pathways may be a promising approach for the maintenance of cartilage integrity and homeostasis.

Oxidative stress is increasingly recognized as a component of OA pathology and is characterized by the excess generation of reactive oxygen species (ROS) [9]. Studies have demonstrated that elevated levels of ROS can promote the production of MMPs and apoptosis in chondrocytes, resulting in the 
destruction of the cartilage matrix in OA $[9,10]$. Nuclear factor (erythroid-derived 2)-like $2(\mathrm{Nrf2})$ is a key transcription factor that plays a crucial role in the protection against oxidative stress through regulating the expression of phase II antioxidant enzymes, including heme oxygenase-1 (HO-1) and $\mathrm{NAD}(\mathrm{P}) \mathrm{H}$ quinine oxidoreductase 1 (NQO1). Recently, Nrf2 activation has been identified as a major chondroprotective factor conferring protection against oxidative stress during OA progression. An in vitro study showed that Nrf2 plays a pivotal role in protecting chondrocytes against IL- $1 \beta$-induced oxidative stress and apoptosis [11]. Further, an in vivo study identified that cartilage damage is increased in Nrf2knockout (KO) mouse OA models [12].

Kruppel-like factor 2 (KLF2), also known as lung Kruppel-like factor (LKLF), is a member of the zinc finger family. KLF2 is a transcription factor that orchestrates the expression of a battery of genes involved in a wide variety of inflammatory disease conditions, such as rheumatoid arthritis, atherosclerosis, and chronic kidney disease [13-15]. Previous studies have demonstrated that in monocytes, KLF2 can inhibit the induction of the expression of proinflammatory factors, such as IL- $1 \beta$, TNF- $\alpha$, and IL-8, which also play an important role in OA pathogenesis [16]. It has also been shown that increasing KLF2 levels could be helpful in decreasing the expression of MMPs, such as MMP3 and MMP13, in monocytes, which mediate a cascade of matrix degradation processes resulting in the destruction of cartilage in OA [17]. Importantly, KLF2 has been shown to play a crucial role in protecting against oxidative damage through the activation of the Nrf2/antioxidant-response element (ARE) signaling pathway $[18,19]$. Despite these findings, the functions of KLF2 in chondrocytes and OA pathogenesis have not yet been investigated in detail, although an in vitro study by Yuan et al. suggested that KLF2 regulates the degradation of COL2A1 by suppressing the IL- $1 \beta$-induced expression of MMP13 in chondrocytes [20]. Therefore, the present study was undertaken to examine the chondroprotective effect of KLF2 and investigate its mechanisms in vitro and in vivo.

In this study, we used SW1353 human chondrocytes and rat models of OA to investigate whether KLF2 is associated with OA pathogenesis. We identified that KLF2 expression is specifically downregulated in human OA cartilage. We then evaluated the functions of KLF2 in OA pathogenesis and examined the potential mechanisms in vitro. We found that pharmacological or lentiviral overexpression of KLF2 preserves cartilage homeostasis by suppressing apoptosis and MMP expression through the negative regulation of ROS. Furthermore, our data demonstrate that this chondroprotective effect of KLF2 is mediated, at least in part, through the promotion of Nrf2 nuclear translocation. Overall, our gain-of-function (adenovirus-mediated overexpression of KLF2) approach in rat knee joint tissue clearly indicates that KLF2 is a potential therapeutic target for the treatment of osteoarthritis.

\section{Materials and Methods}

2.1. Human Articular Cartilage Samples. This study was reviewed and approved by the Institutional Review Board
(IRB) of China Medical University. All patients involved in this study gave informed consent. Human OA cartilage samples with obvious erosion were obtained from patients undergoing total knee arthroplasty $(n=6)$. Normal cartilage samples without gross signs of degradation were obtained from patients undergoing total hip replacement for femur neck fracture $(n=6)$. Paired smooth and damaged cartilage was obtained from the same OA patients undergoing total knee arthroplasty $(n=10)$. The difference between the smooth (relatively healthy area) and damaged (severely damaged area) articular surfaces was distinguished using the Mankin scoring system [21]. Another 24 cartilage samples were obtained from 24 patients with different degrees of OA according to the Kellgren-Lawrence scale system ( $n=6$ per group). After surgical harvesting, the cartilage samples were immediately stored at $-80^{\circ} \mathrm{C}$ for mRNA extraction or fixed in $4 \%$ paraformaldehyde for histological and immunohistochemical analyses.

2.2. SW1353 Human Chondrocyte Culture and Treatment. SW1353 human chondrocytes (American Type Culture Collection, Manassas, VA, USA) were seeded on 6-well plates and cultured in DMEM/high-glucose (HyClone, Logan, UT) containing $10 \%$ FBS (HyClone) and $1 \%$ penicillin/streptomycin (Solarbio, Beijing, China) at $37^{\circ} \mathrm{C}$ in humidified air with $5 \% \mathrm{CO}_{2}$, and the medium was replaced every 2 days. At approximately $80 \%$ confluence, the cells were serum starved overnight and then stimulated with $20 \mathrm{ng} / \mathrm{ml} \mathrm{IL-} 1 \beta$ (R\&D Systems), $10 \mu \mathrm{M}$ simvastatin (Calbiochem), $10 \mu \mathrm{M}$ GGPP (Sigma), and $5 \mu \mathrm{M}$ ML385 (MCE) when appropriate. The concentrations were derived from previously published articles [11, 22-24].

2.3. Cell Viability Assay. Cell viability was determined with a cell counting kit-8 (CCK-8; Beyotime Biotechnology) according to the manufacturer's instructions. In brief, SW1353 cells were plated in 96-well plates at a density of 2000 cells/well. At the appropriate time point, $10 \mu \mathrm{l}$ of CCK- 8 solution was added to each well and the plate was incubated at $37^{\circ} \mathrm{C}$ for $2 \mathrm{~h}$. Subsequently, the absorbance of each well at $450 \mathrm{~nm}$ was measured.

2.4. Real-Time Reverse Transcription-Polymerase Chain Reaction. After the appropriate treatment, total RNA was isolated from SW1353 cells or cartilage tissues using TRIzol reagent (Life Technologies Corporation, USA) following the manufacturer's instructions. Single-stranded cDNA was synthesized from purified RNA using a RevertAid first-strand cDNA synthesis kit (Thermo Fisher Scientific, USA) in accordance with the manufacturer's instructions. cDNA was used for real-time PCR analysis using a SYBR ${ }^{\circledR}$ Premix Ex TaqTM kit (Takara Bio, China) on an ABI 7500 Fast Real-Time PCR system (Applied Biosystems, USA) according to the manufacturer's instructions. Primers were synthesized by Sangon Biotech (China). All samples were analyzed in triplicate. The mRNA value for the target gene was determined using the $2^{-\Delta \Delta \mathrm{Ct}}$ method.

2.5. Western Immunoblotting and Immunoprecipitation. After receiving the indicated treatments, SW1353 cells 
were washed with cold phosphate-buffered saline (PBS). Total proteins were extracted using RIPA lysis buffer (Beyotime) with $1 \mathrm{mM}$ phenylmethanesulfonyl fluoride (PMSF, Beyotime). Cytosolic and nuclear proteins were extracted by a Nuclear and Cytoplasmic Protein Extraction kit (Beyotime) according to the manufacturer's instructions. Protein concentrations were calculated using a BCA Protein Assay kit (Beyotime). Samples containing equivalent amounts of lysate protein $(20 \mu \mathrm{g})$ were separated on sodium dodecyl sulfate-polyacrylamide gels (SDS-PAGE) and transferred to a PVDF membrane (Bio-Rad, USA). After blocking in 5\% nonfat milk for $2 \mathrm{~h}$ at room temperature, the membranes were incubated with primary antibodies in TBST overnight at $4^{\circ} \mathrm{C}$. Antibodies against KLF2 were purchased from Abcam. Antibodies against MMP3, MMP9, MMP13, COL2A1, Nrf2, HO-1, NQO1, and $\beta$-actin were purchased from Proteintech. Thereafter, the membranes were washed with TBST and then incubated with a secondary antibody (HRP-conjugated AffiniPure goat anti-rabbit IgG, Proteintech) for $2 \mathrm{~h}$ at room temperature. The blots were visualized by enhanced chemiluminescence (ECL, Beyotime). The densities of the protein bands were quantified using ImageJ (http://rsb. info.nih.gov/ij/, Bethesda, MD, USA). For immunoprecipitation, cells were overexpressed with KLF2 using lentivirus. After that, the cells were rinsed with phosphate-buffered saline, scraped into lysis buffer $(150 \mathrm{mM} \mathrm{NaCl}, 50 \mathrm{mM}$ Tris- $\mathrm{HCl}$ at $\mathrm{pH} 7.5,5 \mathrm{mM}$ EDTA, $1 \mathrm{mM}$ phenylmethylsulfonyl fluoride, and $0.5 \%$ Nonidet P-40), and placed on ice for $30 \mathrm{~min}$. Cells were then spun at $15,000 \mathrm{~g}$ for $15 \mathrm{~min}$, and the supernatant was incubated overnight at $4^{\circ} \mathrm{C}$ with either anti-KLF2, anti-Nrf2 antibody, or control IgG at $4^{\circ} \mathrm{C}$ and protein A/G agarose resin for overnight. The samples were washed in lysis buffer for five times and then were used for immunoblot analysis.

2.6. Immunofluorescence Staining. After receiving the indicated treatments, the SW1353 cells were washed twice with PBS and fixed in $4 \%$ formaldehyde for $15 \mathrm{~min}$. After being washed three times with PBS, the cells were treated with $0.2 \%$ Triton X-100 at $37^{\circ} \mathrm{C}$ for $20 \mathrm{~min}$. The cells were then washed three times with PBS (5 min each) and blocked with $5 \%$ bovine serum albumin (BSA) at $37^{\circ} \mathrm{C}$ for $1 \mathrm{~h}$. After washing with PBS, KLF2 (Abcam), Nrf2 (Proteintech), and MMP13 (Proteintech) antibodies $(1: 200)$ were added for overnight incubation at $4^{\circ} \mathrm{C}$. An Alexa Fluor 488 or 594labeled secondary antibody $(1: 200)$ (Proteintech) was then added for $30 \mathrm{~min}$ incubation at $37^{\circ} \mathrm{C}$. Following washing with PBS, $0.1 \%$ DAPI (Beyotime) was added for $10 \mathrm{~min}$ of staining. The slides were observed using fluorescence microscopy (Olympus).

2.7. Measurement of Reactive Oxygen Species (ROS) Levels. After cells received the indicated treatments, the level of intracellular ROS was measured using a Reactive Oxygen Species Assay kit (Beyotime). Briefly, SW1353 cells were harvested and stained with $10 \mu \mathrm{M}$ DCF-DA in the dark at $37^{\circ} \mathrm{C}$ for $15 \mathrm{~min}$. The cells were then rinsed 3 times with PBS and immediately detected using a flow cytometer $(525 \mathrm{~nm})$.
2.8. Measurement of Apoptosis. After receiving the indicated treatments, the SW1353 cells were collected and suspended in binding buffer. Then, the cells were stained with Annexin V-FITC/propidium iodide (PI) for $15 \mathrm{~min}$ in the dark according to the manufacturer's instructions (Becton Dickinson). Stained cells were detected by flow cytometry and analyzed using FlowJo software. Apoptosis of chondrocytes in rat cartilage tissue was detected using a terminal deoxynucleotidyl transferase dUTP nick-end labeling (TUNEL) assay kit (Beyotime) according to the manufacturer's instructions.

2.9. Lentivirus-Mediated Overexpression of KLF2. Human KLF2 and nonspecific control lentiviruses were purchased from Genechem (Shanghai, China). SW1353 cells were transfected with lentivirus in accordance with the manufacturer's instructions. KLF2 overexpression was confirmed by realtime PCR and Western blotting.

2.10. Animal OA Model and Adenovirus-Mediated Overexpression of KLF2 in Rat Knee Joint Tissue. Male Sprague-Dawley rats ( 8 weeks old, $210 \pm 10 \mathrm{~g}$ in weigh, and specifically pathogen-free) were used in the present study. All rats were housed with a $12 \mathrm{~h}$ light/dark cycle at a constant room temperature $\left(25^{\circ} \mathrm{C}\right)$ with free access to food and water. Experimental OA was induced in 8-week-old male rats by intra-articular (IA) injection of monoiodoacetate (MIA; $1 \mathrm{mg}$ per cavity in $50 \mu \mathrm{l}$ of sterile saline). The control group received an IA injection of $50 \mu \mathrm{l}$ of sterile saline. Adenovirus-KLF2 (Ad-KLF2) and a control virus (Ad-C) were used to overexpress KLF2 in the rat knee joint. After injection of MIA or sterile saline on day 3, Ad-KLF2 or Ad-C was injected into the knee joints of rats for three consecutive weeks $\left(1 \times 10^{9}\right.$ plaque-forming units (PFUs) in a total volume of $10 \mu \mathrm{l}$ ), as indicated in a previous study [25]. ML385 was used to inhibit Nrf2 expression in the rat knee joint. After the first IA injection of Ad-KLF2 on day 3, rat received an IA injection of ML385 $(10 \mu \mathrm{M}, 20 \mu \mathrm{l}$ per joint) two times a week for 4 weeks. Rats were sacrificed 6 weeks after injection of MIA or sterile saline and subjected to histological analyses.

2.11. Histopathologic Analysis. Human knee articular samples and rat knee joints were prepared and fixed in $4 \%$ paraformaldehyde. Then, the samples were decalcified in $10 \%$ EDTA for 21 days and embedded in paraffin. Tissue sections $(5 \mu \mathrm{m})$ were stained with safranin $\mathrm{O} /$ fast green following standard protocols to determine cartilage degradation under light microscopic examination. The Osteoarthritis Research Society International (OARSI) scoring system was used to assess joint cartilage degeneration [26]. Because both the tibial and femoral cartilages were assessed in the present study, the maximum OARSI score was 48. Three independent investigators who were blinded to the experimental groups performed the scoring. Immunohistochemistry was further performed to analyze the protein expression of KLF2, MMP13, and COL2A1 in histological sections of human knee articular samples and rat knee joints. Primary antibodies against KLF2 (Abcam), MMP13, and COL2A1 (Proteintech) were used at $1: 100-1: 200$ dilutions and incubated overnight at $4^{\circ} \mathrm{C}$. Then, the sections were incubated with a 
biotinylated secondary antibody. The reaction was developed using a DAB kit (BD Bioscience, Franklin Lakes, NJ, USA). The expression of KLF2 and MMP13 was evaluated by calculating the percentage of immunopositive cells. The expression of COL2A1 was evaluated by calculating the relative intensity.

2.12. Statistical Analysis. Each experiment was repeated three times using three independent samples, and the data were expressed as the mean \pm standard deviation. The $n$-value indicates the number of human specimens or rats per group. All statistical analyses were performed using GraphPad Prism software. For cell-based in vitro studies, statistical significance was analyzed by means of unpaired Student's $t$-test for comparisons between two groups and one-way ANOVA with post hoc test for multiple comparisons. For rat-based in vivo studies, the nonparametric Mann-Whitney $U$ test was used. The Pearson $\chi^{2}$ test was used to analyze the relationship between KLF2 and COL2A1 expression levels. A $p$ value $<0.05$ was considered statistically significant.

\section{Results}

3.1. KLF2 Expression Is Downregulated in Human OA Cartilage. To assess the possible association of KLF2 with OA pathogenesis, we first downloaded (https://www.ncbi .nlm.nih.gov/gds/) and analyzed the GSE114007 microarray data, which include 18 normal and 20 OA human knee cartilage tissues. The cartilage tissues in GSE114007 were obtained from the weight-bearing regions on the medial and lateral femoral condyles. The results from the microarray analysis showed that KLF2 expression was obviously decreased in OA cartilage tissues compared to normal cartilage tissues (Figure 1(a)). To further validate this finding, we collected 6 pairs of OA cartilage tissues from patients undergoing total knee arthroplasty and normal cartilage tissues from patients with femoral neck fractures. Real-time PCR was used to detect the mRNA expression of KLF2, MMP13, and COL2A1. The results indicated that compared to that in normal cartilage tissues, KLF2 expression in OA cartilage tissues was decreased (Figure 1(b)). As expected, the expression of the osteoarthritic marker MMP13 was significantly increased, and COL2A1 expression was significantly reduced in articular cartilage in the OA samples (Figure 1(b)). Histologic changes between normal and OA samples were explored by safranin $\mathrm{O} /$ fast green staining. Severe cartilage loss was observed in OA cartilage tissues (reduced safranin O staining) (Figure 1(c)). Consistent with the mRNA level results, the immunostaining results revealed that compared to normal cartilage tissues, OA cartilage tissues displayed decreased expression of KLF2 and COL2A1 and high expression levels of MMP13, reflecting cartilage degradation (Figures 1(c) and 1(d)). To further support the above results, we next explored the expression pattern of KLF2 in the smooth and damaged cartilage samples from the same OA patient. The mRNA expression of KLF2 in the damaged cartilage tissue was considerably decreased compared with that in the smooth cartilage tissue from the same patient (Figure $1(\mathrm{e})$ ). We next explored the correlation between the expression levels of KLF2 and the severity of knee OA. The severity of knee OA was determined by the Kellgren-Lawrence scale system [27], which is a common method of classifying the severity of knee OA using five grades (0-4) (Figure 1(f)). The expression levels of KLF2 and COL2A1 were detected using real-time PCR. Our results showed that both KLF2 and COL2A1 mRNA levels gradually decreased as the grade decreased from grade 1 to grade 4 (Figure 1(g)). Pearson's correlation analysis was used to explore the correlation between KLF2 and COL2A1 expression levels. The results indicated a significant correlation between the expression level of KLF2 and COL2A1 (Pearson's $R$ value $=0.8647, p<0.05$ ). These clinical results collectively indicate that KLF2 may be involved in the pathogenesis of OA.

\subsection{KLF2 Expression Is Downregulated in SW1353 Human} Chondrocytes during IL-1 $\beta$-Induced OA Pathogenesis. Accumulating evidence indicates that interleukin- $1 \beta$ (IL-1 $\beta$ ) is a proinflammatory cytokine that plays a critical role in the pathogenesis of OA $[28,29]$. To test the influence of proinflammatory cytokines on the expression of KLF2, we examined the effect of IL- $1 \beta$ on the expression of KLF2 in SW1353 human chondrocytes by real-time PCR and Western blot analysis. IL- $1 \beta$ caused dose- and time-dependent decreases in KLF2 mRNA and protein levels in SW1353 human chondrocytes (Figures 2(a) and 2(b)). We also measured KLF2 protein levels by immunofluorescence staining, which indicated decreased KLF2 expression in IL- $1 \beta$-treated cells $(20 \mathrm{ng} / \mathrm{ml}, 24 \mathrm{~h})$ and confirmed that KLF2 was localized in the nuclei of SW 1353 cells (Figure 2(c)). The immunofluorescence staining results also showed that IL- $1 \beta$ treatment significantly decreased COL2A1 protein expression levels and increased MMP13 protein expression levels in chondrocytes, which indicates that IL- $1 \beta$ stimulation successfully mimics OA pathogenesis in vitro (Figures $2(\mathrm{~d})$ and $2(\mathrm{e})$ ). Altogether, these results further confirm that KLF2 may be involved in the pathogenesis of $\mathrm{OA}$ in vitro.

3.3. KLF2 Protects SW1353 Cells from IL-1 $\beta$-Induced Apoptosis and Matrix Degradation through the Suppression of ROS Production. Since apoptosis of chondrocytes is considered a central feature in OA progression [30], we investigated the effects of KLF2 overexpression on IL- $1 \beta$-induced apoptosis in SW1353 cells using pharmacological and lentiviral strategies. As shown in Figures 3(a) and 3(b), KLF2 expression was significantly increased at the mRNA and protein levels by specific KLF2 overexpression using lentivirus or treatment with the KLF2 activator simvastatin [31], whereas it was significantly decreased by treatment with the KLF2 inhibitor GGPP. Our CCK- 8 results showed that IL- $1 \beta$ treatment significantly decreased SW1353 cell viability, whereas genetic or pharmacological overexpression of KLF2 rescued the IL- $1 \beta$-induced decrease in cell viability (Figure $3(\mathrm{c})$ ). To further explore the protective role of KLF2, KLF2overexpressing cells were treated with the KLF2-specific inhibitor GGPP. As shown in Figure 3(c), GGPP abrogated the effects of KLF2 overexpression on cell viability. Consistent with these findings, genetic or pharmacological 

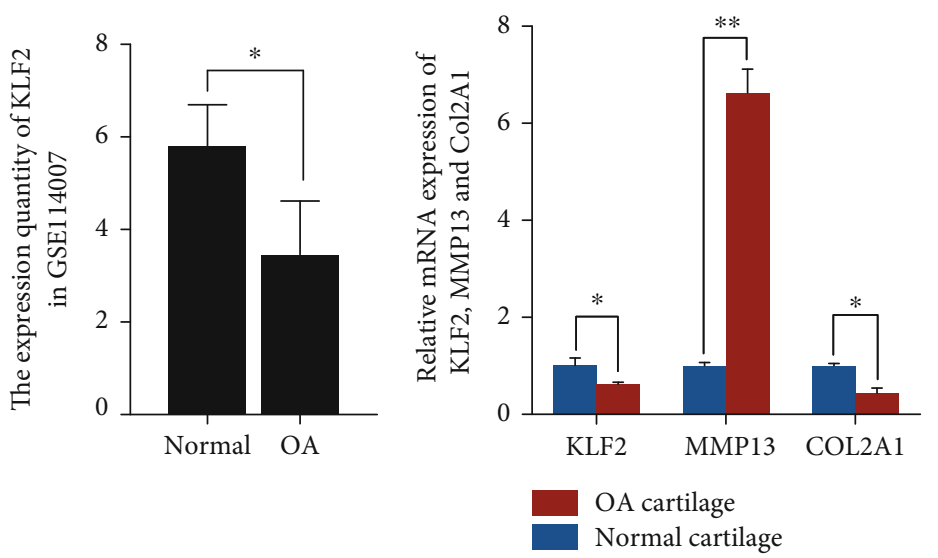

(a)

(b)
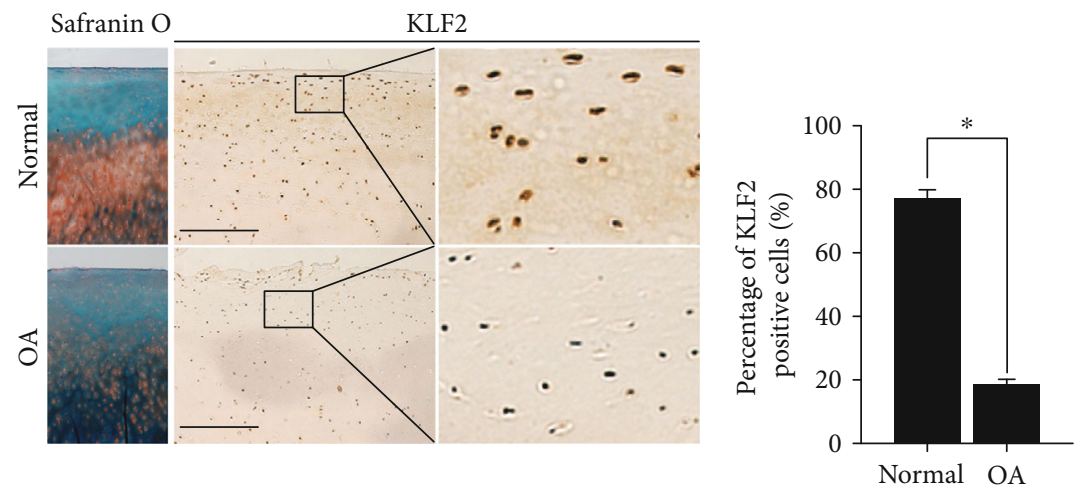

(c)
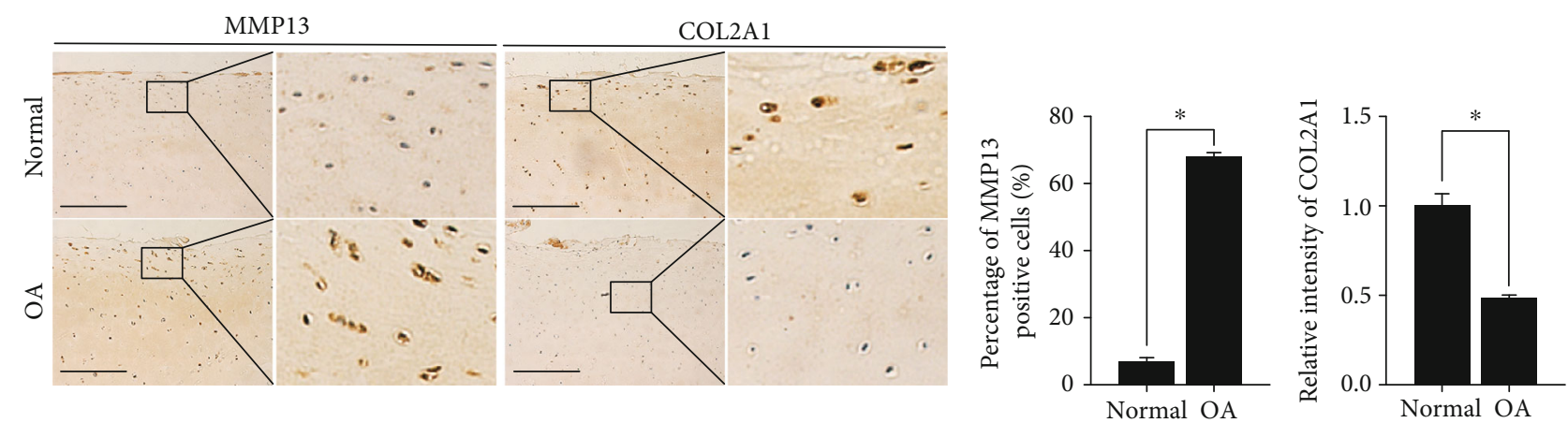

(d)

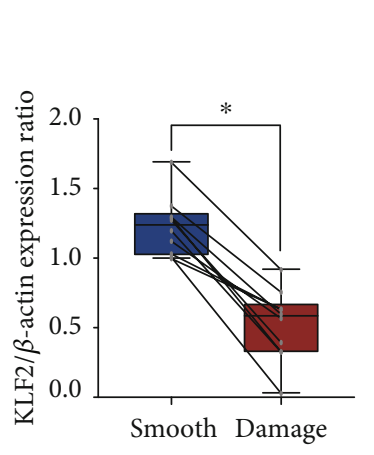

(e)
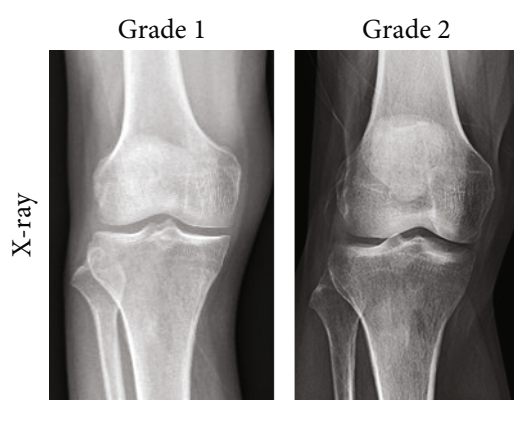

(f)

Figure 1: Continued. 

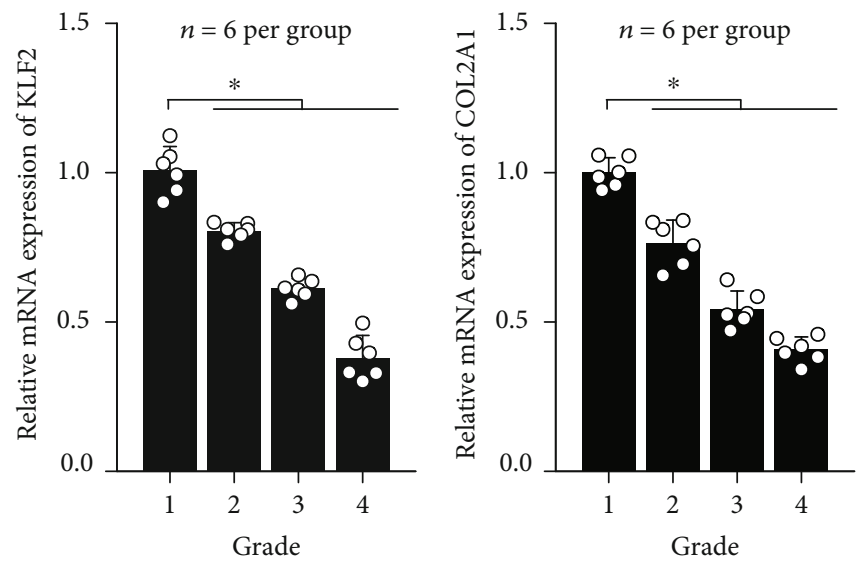

(g)

FIgURE 1: KLF2 expression is suppressed during osteoarthritis pathogenesis. (a) Microarray analysis of KLF2, MMP13, and COL2A1 expression in OA cartilage $(n=20)$ and normal cartilage $(n=18)$ based on GSE114007. (b) Real-time PCR analysis was used to assess the mRNA levels of KLF2 in normal and OA cartilage from human patients ( $n=6$ per group). (c) Representative images of safranin O/fast green staining and immunohistochemical staining with antibodies against KLF2 in normal and OA cartilage from human knee joints ( $n=6$ per group). The scale bar represents $200 \mu \mathrm{m}$. The right panels show the quantification of KLF2 immunohistochemical staining. (d) Representative images of immunohistochemical staining with antibodies against MMP13 and COL2A1 in normal and OA cartilage from human knee joints ( $n=6$ per group). The scale bar represents $200 \mu \mathrm{m}$. The right panels show the quantification of the immunohistochemical staining of MMP13 and COL2A1. (e) RNA was isolated from smooth and damaged regions of OA cartilage from the same patient $(n=10)$, and the levels of KLF2 mRNA were evaluated by real-time PCR. (f) Representative X-rays of different stages of OA graded by the Kellgren-Lawrence scale system. (g) RNA was isolated from grade 1-4 OA patients, and KLF2 mRNA expression was evaluated by real-time PCR ( $n=6$ per group). All data are expressed as the mean \pm SD. ${ }^{*} p<0.005$ and ${ }^{* *} p<0.0005$.

overexpression of KLF2 significantly decreased the percentage of IL- $1 \beta$-induced apoptotic SW1353 cells, while the addition of the KLF2 inhibitor GGPP abrogated the effects of KLF2 overexpression on SW1353 apoptosis (Figure 3(d)).

Since the degradation of articular cartilage is a hallmark of OA pathogenesis, we next explored the effects of KLF2 overexpression on IL- $1 \beta$-induced cartilage degradation in SW1353 cells. We measured the expression of MMP3, MMP9, MMP13, and COL2A1 (the primary component of ECM) in SW1353 cells with and without KLF2 overexpression and stimulated with IL-1 $\beta$. Our results indicated that genetic or pharmacological overexpression of KLF2 in conjunction with IL-1 $\beta$ stimulation significantly reduced MMP3, MMP9, and MMP13 expression and increased COL2A1 expression in SW1353 cells; furthermore, the addition of the KLF2 inhibitor GGPP abrogated the effects of KLF2 overexpression (Figures 3(e) and 3(f)).

Oxidative stress is increasingly recognized as being closely associated with OA pathology, and accumulating evidence has demonstrated that elevated levels of ROS lead to apoptosis and ECM degradation in OA pathogenesis [5]. To determine whether the chondroprotective effects of KLF2 overexpression are due to attenuated ROS production, we monitored the extent of ROS production in SW 1353 cells. Our results showed that IL-1 $\beta$ stimulation significantly increased the levels of ROS in SW1353 cells, whereas genetic or pharmacological overexpression of KLF2 in SW1353 cells suppressed the IL- $1 \beta$-induced generation of ROS (Figure 3(g)). This effect was partially weakened by the addition of the KLF2 inhibitor GGPP (Figure 3(g)). These results collectively indicate that the overexpression of
KLF2 effectively alleviates IL- $1 \beta$-induced catabolic events and apoptosis through the suppression of ROS generation in chondrocytes.

3.4. KLF2 Overexpression Inhibits Matrix Degradation and SW1353 Cell Apoptosis through the Activation of the Nrf2/ARE Signaling Pathway. The Nrf2/ARE signaling pathway is primarily responsible for cellular defences against oxidative stress under the pathological conditions of OA [11]. To further explore the underlying mechanism of the KLF2mediated suppression of IL- $1 \beta$-induced ROS generation in SW1353 cells, we determined whether the chondroprotective effect of KLF2 is mediated by the activation of the transcription factor Nrf2. As shown in Figures 4(a) and 4(b), the expression of Nrf2 in SW1353 cells was successfully ablated by ML385 (an inhibitor of Nrf2) treatment. We then examined the effect of KLF2 overexpression on the expression of Nrf2 downstream proteins in SW1353 cells. The results showed that overexpression of KLF2 caused a significant increase in NQO1 and HO-1 expression at the mRNA and protein levels, while the addition of the $\mathrm{Nrf} 2$ inhibitor ML385 abrogated the KLF2-induced increase in HO-1 and NQO1 expression (Figures 4(c)-4(e)). To study how KLF2 affects Nrf2 activity during oxidative stress, SW1353 cells were infected with nonspecific control lentiviruses or KLF2 lentiviruses. Western blot analysis of Nrf2 revealed that KLF2 promotes the nuclear translocation of Nrf2 protein in SW1353 cells (Figure 4(f)). Similar results were observed in a parallel immunofluorescence analysis (Figure $4(\mathrm{~g})$ ). This observation is consistent with those of previously published reports in endothelial cells and hepatic stellate cells [18, 23]. To determine whether KLF2 could interact with Nrf2 in cells, 

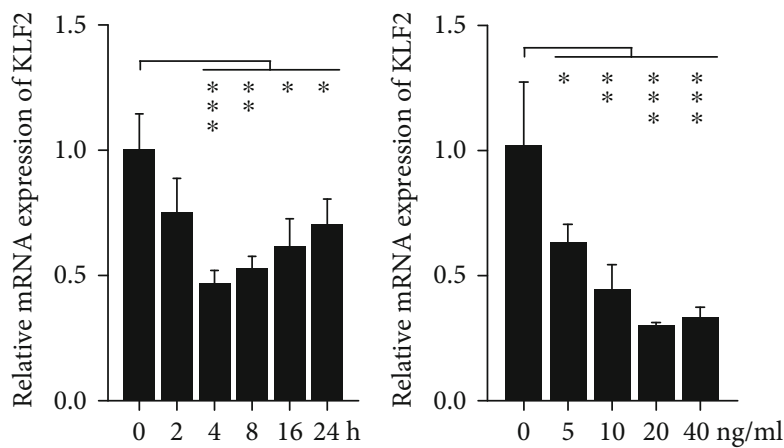

(a)
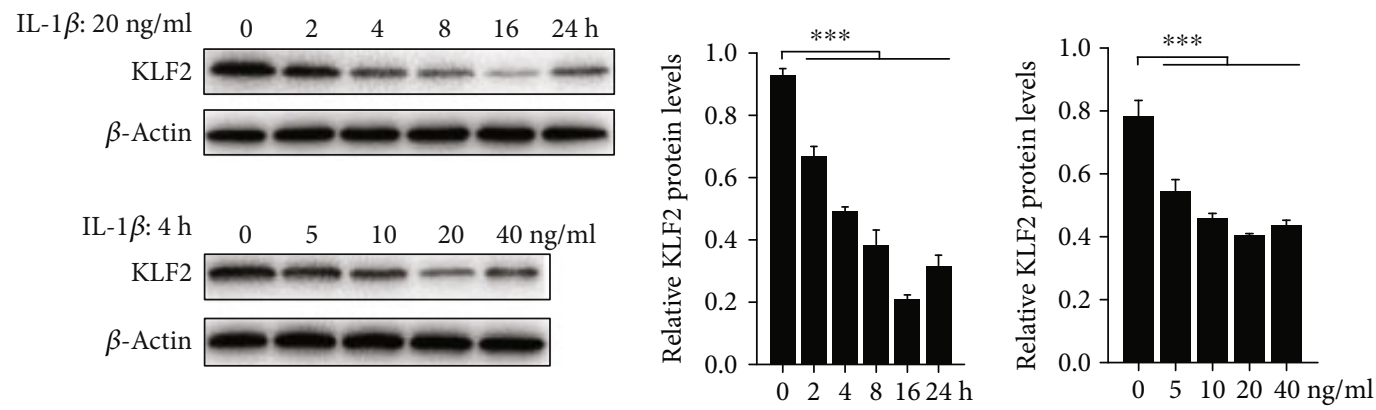

(b)

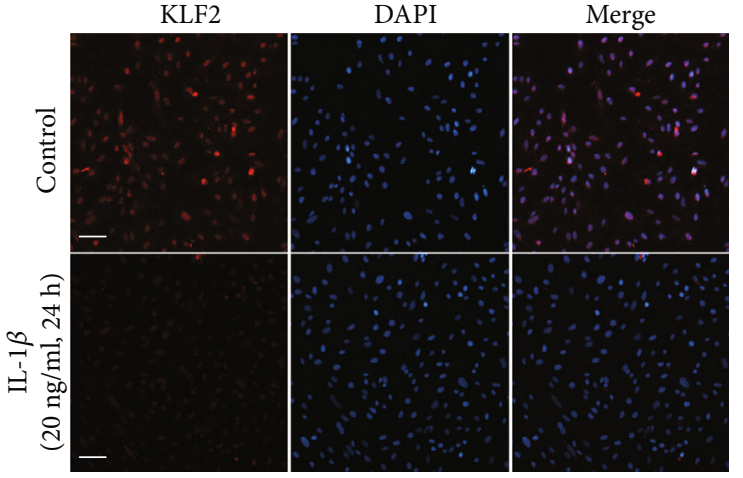

(c)

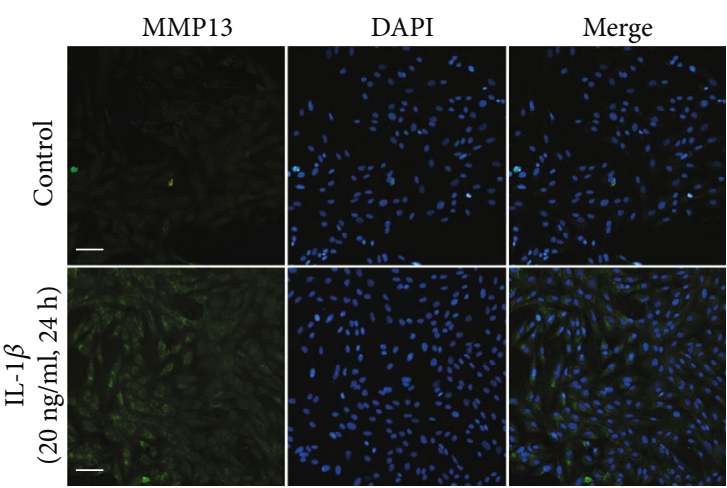

(d)

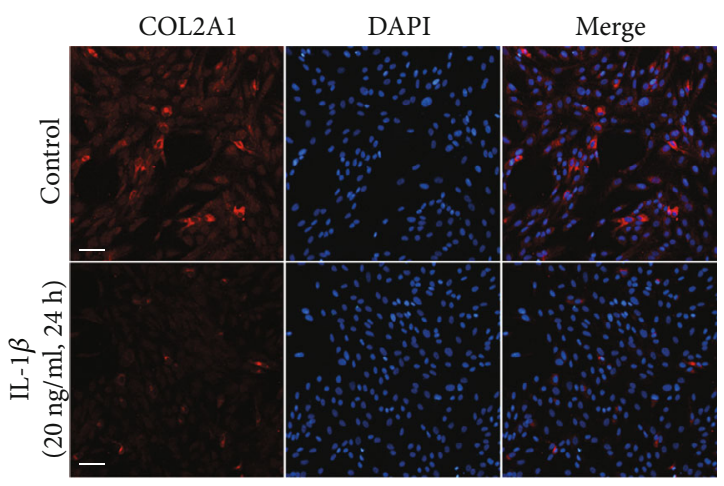

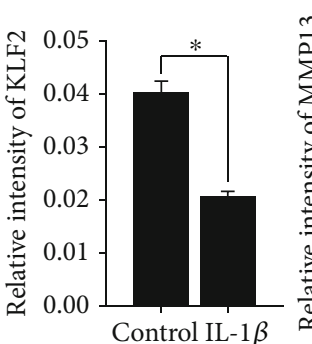
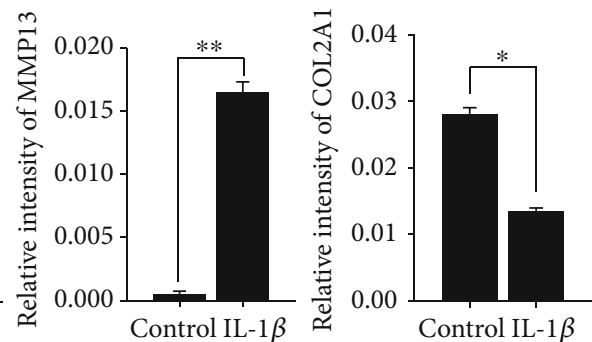

(e)

Figure 2: Expression of KLF2 in SW1353 cells stimulated with IL-1 $\beta$ at different concentrations for various durations. (a, b) SW1353 cells were pretreated with IL- $1 \beta(20 \mathrm{ng} / \mathrm{ml})$ for the indicated times or with IL- $1 \beta(4 \mathrm{~h})$ at the indicated concentration. KLF2 expression was measured by real-time PCR (a) and Western blot analysis (b). $\beta$-Actin was used as an endogenous control. Quantitative analysis of KLF2 protein levels based on the specific signal intensities measured using ImageJ. (c-e) Detection of KLF2, MMP13, and COL2A1 expression in SW1353 cells treated with IL- $1 \beta(20 \mathrm{ng} / \mathrm{ml}, 224)$ by immunofluorescence microscopy. The scale bar represents $50 \mu \mathrm{m}$. All data are expressed as the mean \pm SD. ${ }^{*} p<0.05,{ }^{* *} p<0.005$, and ${ }^{* * *} p<0.0005$. 


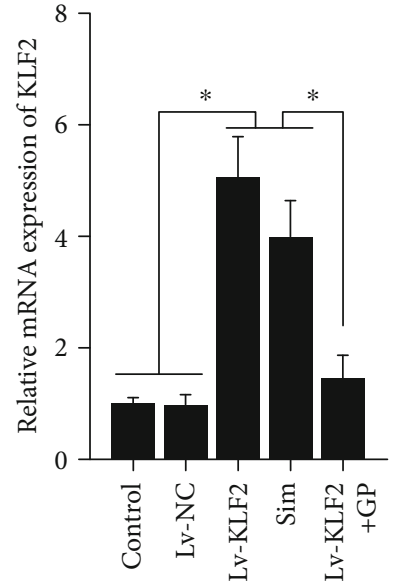

(a)

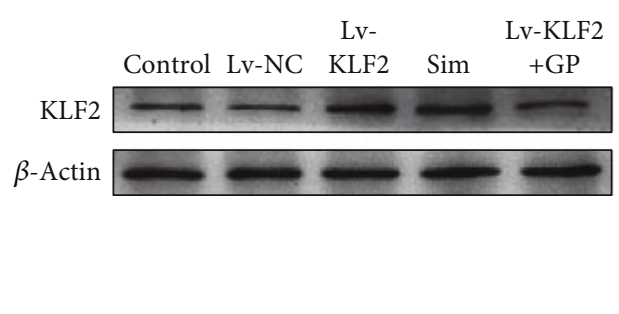

(b)

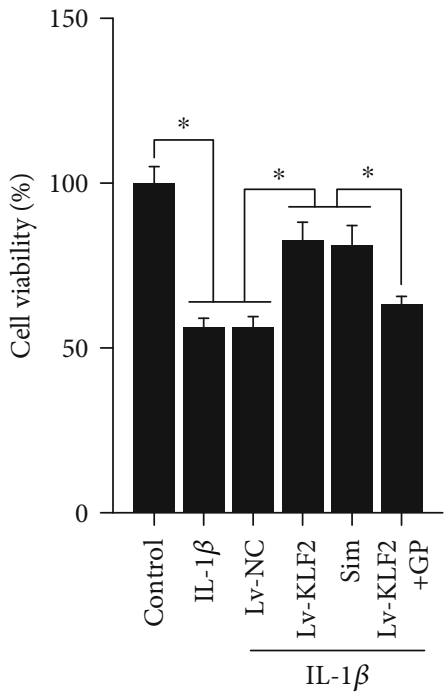

(c)
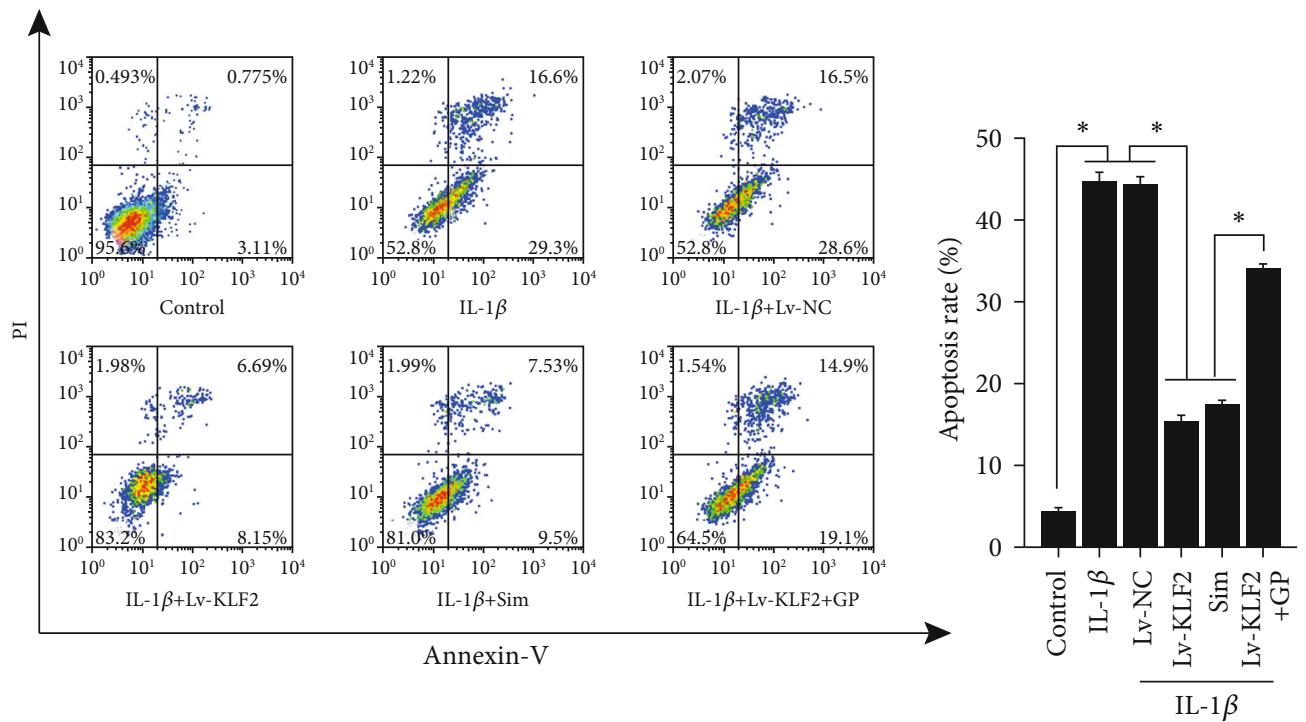

(d)

Figure 3: Continued. 


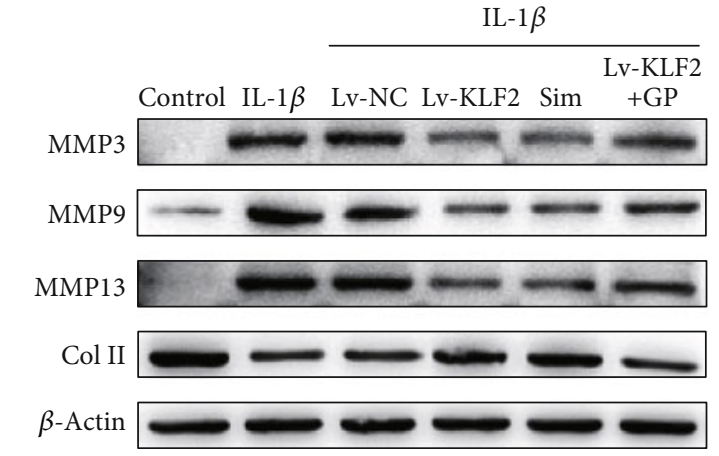

(e)
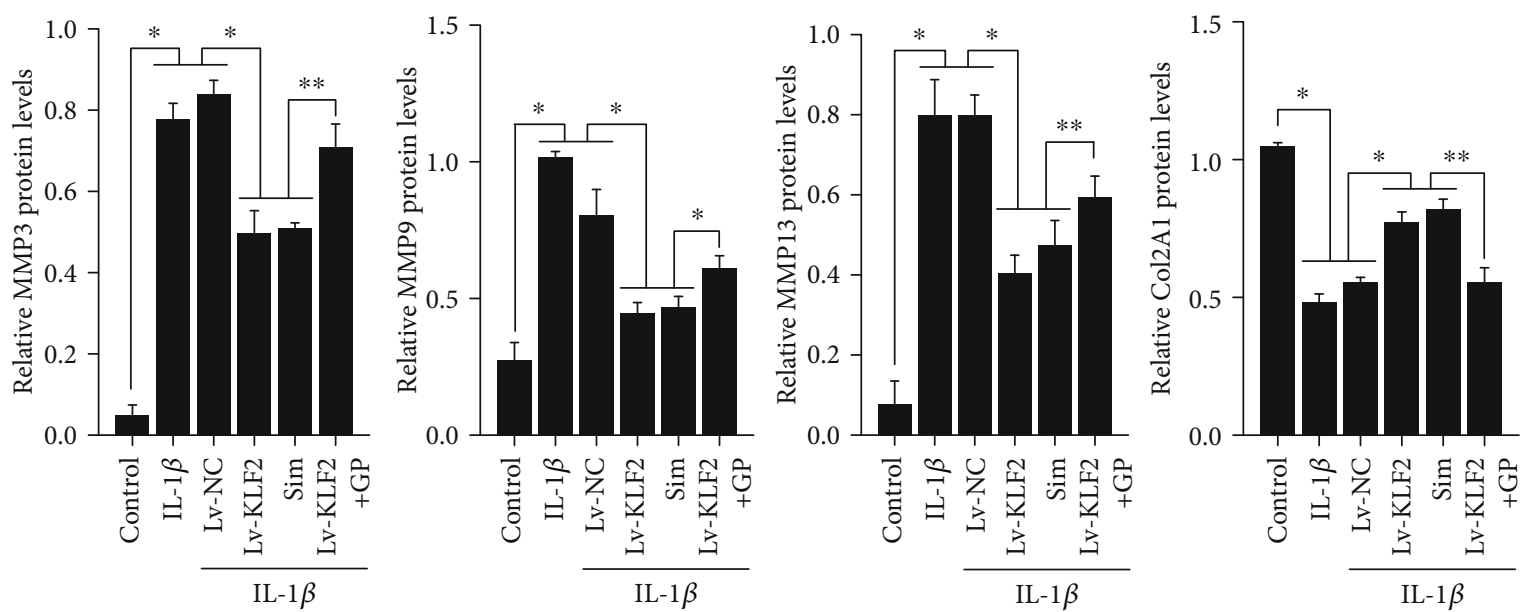

(f)
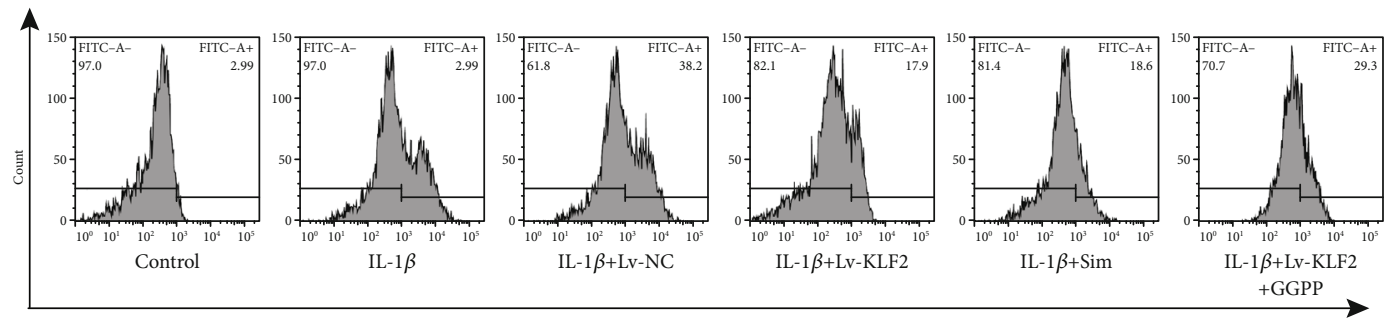

FITC-A

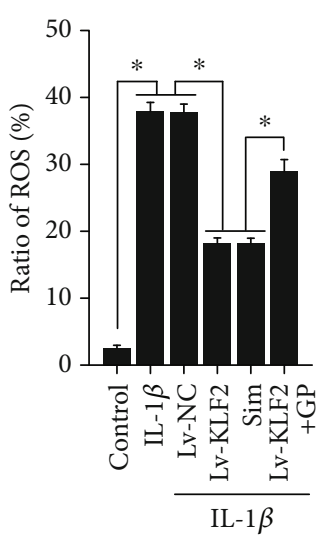

(g)

FIGURE 3: KLF2 overexpression inhibits IL-1 $\beta$-induced apoptosis and chondrocyte degradation through the suppression of ROS production in vitro. SW1353 cells were infected with nonspecific control virus (Lv-NC) or KLF2-expressing virus (Lv-KLF2) or incubated with the pharmacological KLF2 activator simvastatin (Sim). After $24 \mathrm{~h}$, cells were treated with or without GGPP (GP, an inhibitor of KLF2) for $8 \mathrm{~h}$ prior to $24 \mathrm{~h}$ treatment with $20 \mathrm{ng} / \mathrm{ml} \mathrm{IL}-1 \beta$. (a) Real-time PCR was performed to assess KLF2 mRNA expression levels. (b) Western blotting analysis was performed to assess KLF2 expression at the protein level. $\beta$-Actin was used as an endogenous control. Quantitative analysis of KLF2 protein levels based on the specific signal intensities measured using ImageJ. (c) Cell viability was determined by CCK- 8 assay. (d) Apoptosis was determined by Annexin V/PI staining followed by flow cytometry assays. (e) Expression of MMP3, MMP9, MMP13, and COL2A1 was investigated by Western blotting analysis. $\beta$-Actin was used as an endogenous control. (f) Quantitative analysis of MMP3, MMP9, MMP13, and COL2A1 protein levels based on specific signal intensities measured using ImageJ. SW1353 cells were infected with Lv-NC or Lv-KLF2 or incubated with the pharmacological activator of KLF2 simvastatin. After $24 \mathrm{~h}$, cells were treated with or without GP for $8 \mathrm{~h}$ prior to $30 \mathrm{~min}$ treatment with $10 \mathrm{ng} / \mathrm{ml} \mathrm{IL-1 \beta}$. (g) ROS generation was determined by DCF-DA staining followed by flow cytometry analysis. All data are expressed as the mean \pm SD. ${ }^{*} p<0.005$ and ${ }^{* *} p<0.0005$. 

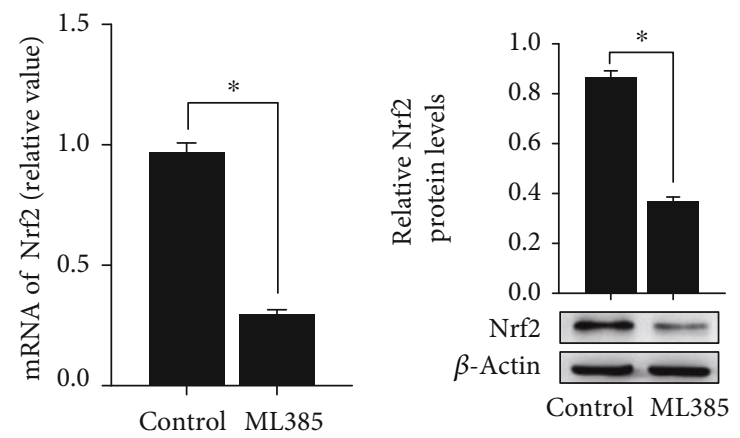

(a)

(b)

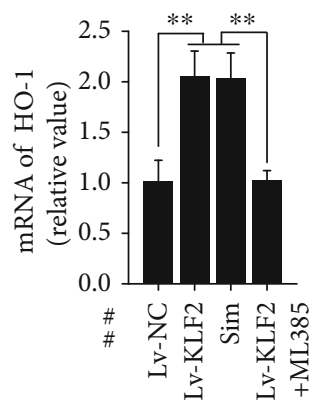

(c)

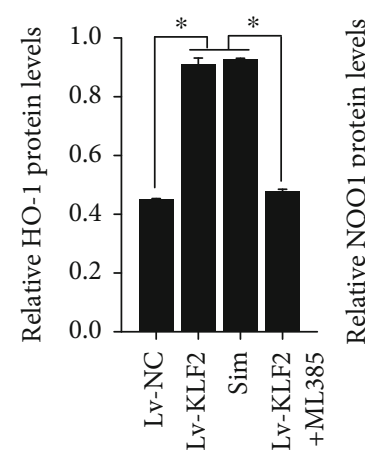

(e)

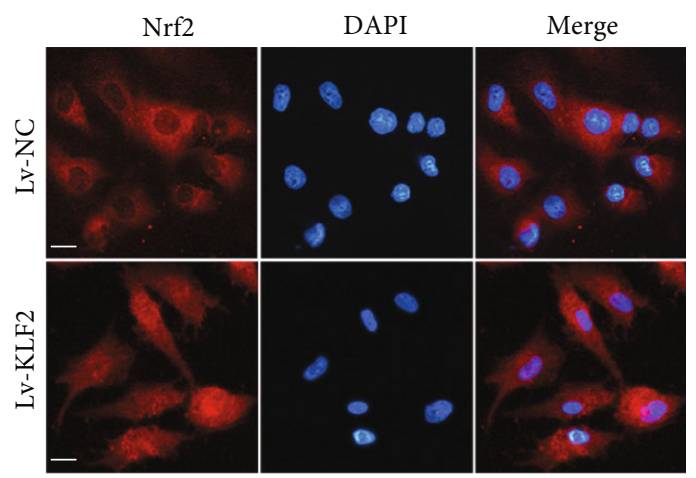

(g)

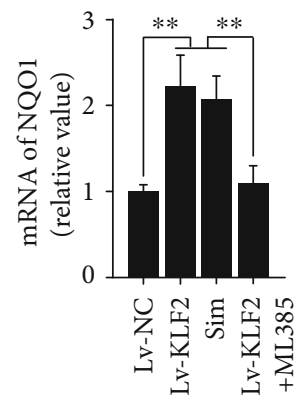

)

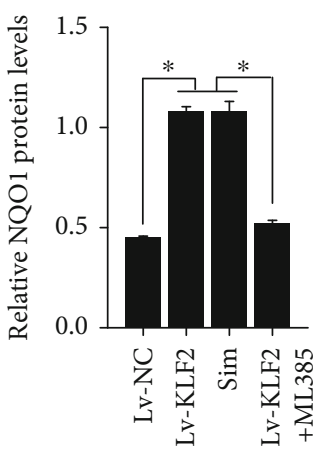

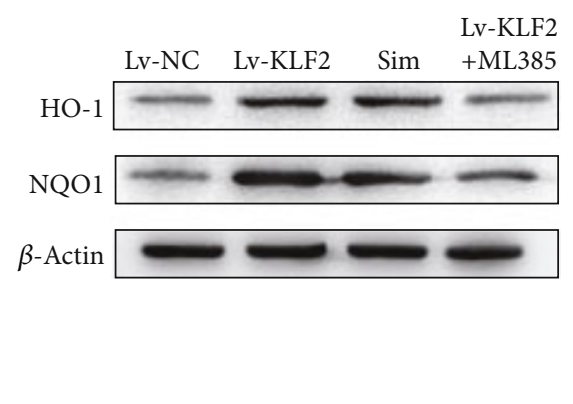

(d)

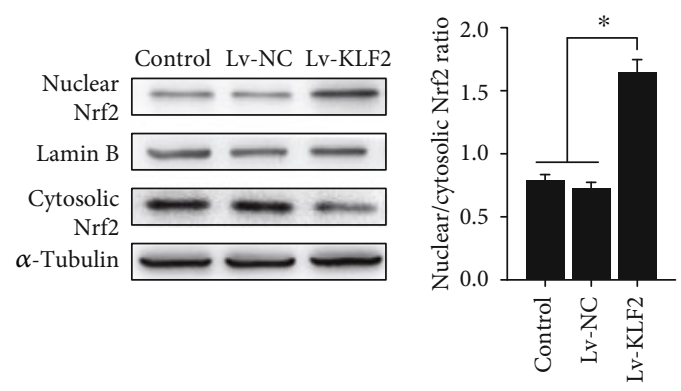

(f)

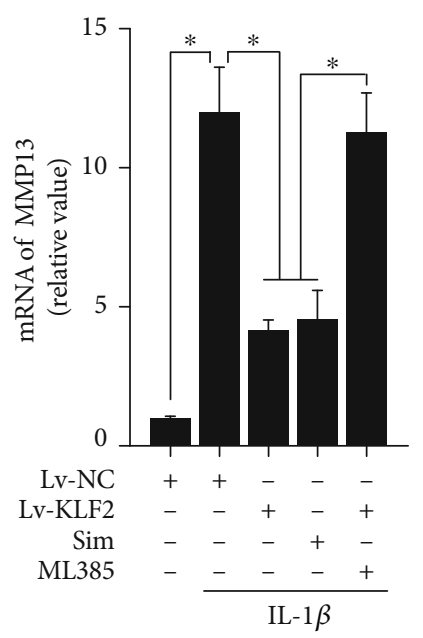

(h)

Figure 4: Continued. 


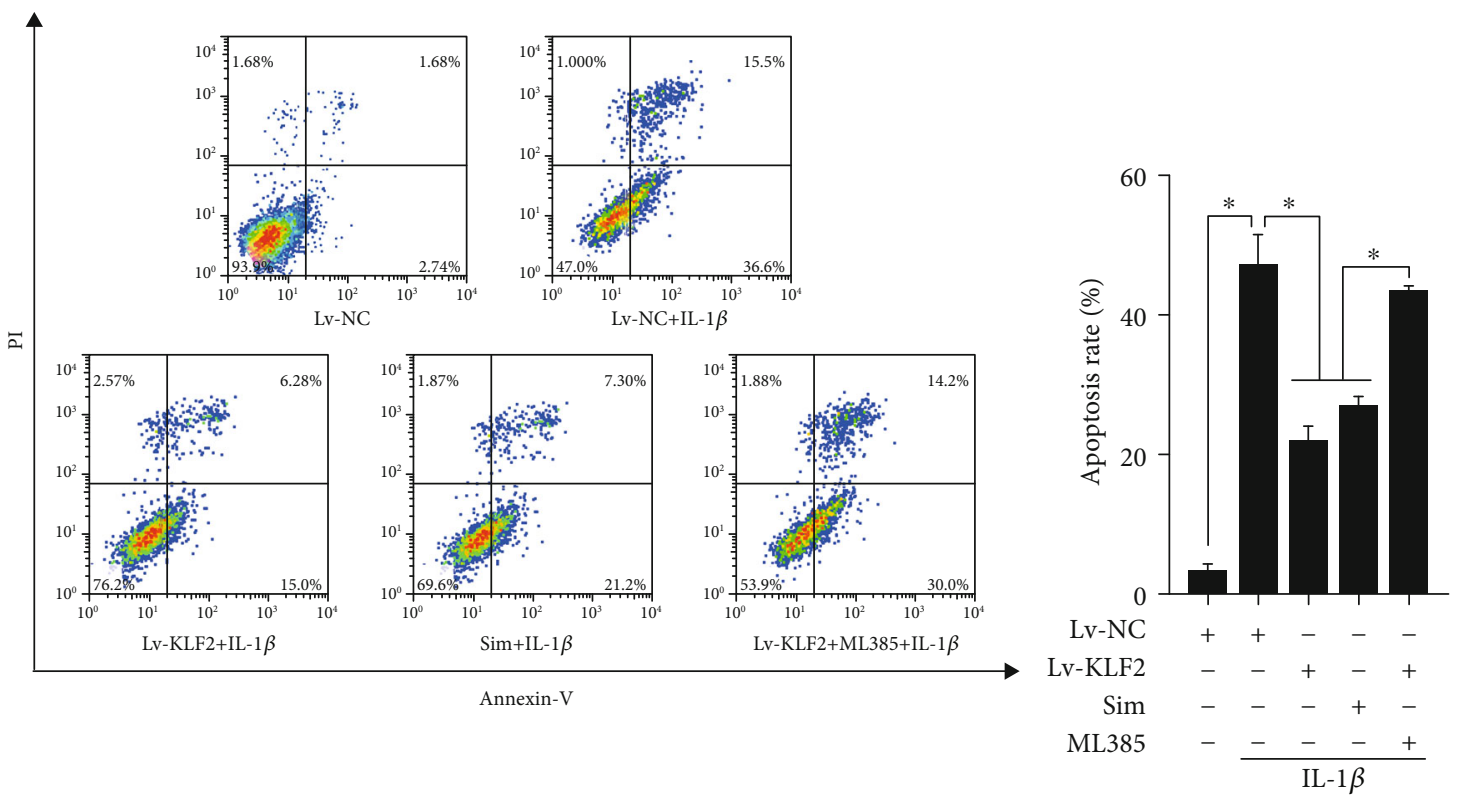

(i)

FIGURE 4: KLF2 exerts chondroprotective effects through the induction of Nrf2 nuclear translocation. (a, b) SW1353 cells were treated with ML385 (an inhibitor of Nrf2). After 12 h, Nrf2 expression was evaluated by real-time PCR (a) and Western blotting analysis (b). $\beta$-Actin was used as an endogenous control. Quantitative analysis of Nrf2 protein levels based on specific signal intensities measured using Image. (c-e) SW1353 cells were infected with nonspecific control virus (Lv-NC) or a KLF2-expressing virus (Lv-KLF2) or incubated with the pharmacological activator of KLF2 simvastatin (Sim). After $24 \mathrm{~h}$, cells were treated with or without ML385 for $12 \mathrm{~h}$. HO-1 and NQO1 mRNA levels were evaluated by real-time PCR (c), and protein levels were evaluated by Western blot analysis (d). $\beta$-Actin was used as an endogenous control. Quantitative analysis of HO-1 and NQO1 protein levels based on specific signal intensities measured using ImageJ (e). (f, g) SW1353 cells were infected with Lv-NC or Lv-KLF2. Nrf2 protein levels in the cytosolic and nuclear fractions were evaluated by Western blotting analysis (f). $\alpha$-Tubulin (cytosolic) and Lamin B (nuclear) were used as endogenous controls. Quantitative analysis of Nrf2 protein levels based on specific signal intensities measured using ImageJ. (g) Immunohistochemical staining of the subcellular localization of Nrf2 protein (red). Nuclei were stained with DAPI (blue). The scale bars represent $10 \mu \mathrm{m}$. (h, i) SW1353 cells were infected with Lv-NC or Lv-KLF2 or incubated with simvastatin. After $24 \mathrm{~h}$, cells were treated with or without ML385 for $12 \mathrm{~h}$ prior to $24 \mathrm{~h}$ of treatment with $20 \mathrm{ng} / \mathrm{ml}$ IL-1 $\beta$. MMP13 mRNA levels were evaluated by real-time PCR (h), and apoptosis was determined by Annexin V/PI staining followed by flow cytometry (i). All data are expressed as the mean \pm SD. ${ }^{*} p<0.005$ and ${ }^{* *} p<0.0005$.

immunoprecipitation was performed. However, the results showed that the protein-protein interaction between KLF2 and Nrf2 was undetected in vitro (Fig. S1). It is likely that KLF2-mediated promotion of Nrf2 nuclear translocation is indirectly enhanced by other mechanisms rather than interacting with each other at the protein level in human articular chondrocytes. To further demonstrate that the chondroprotective effect of KLF2 is Nrf2-dependent, we inhibited Nrf2 expression using ML385. The results showed that Nrf2 inhibition significantly abrogated the KLF2-mediated suppression of MMP13 and apoptosis induced by IL-1 $\beta$ in SW1353 cells (Figures $4(\mathrm{~h})$ and $4(\mathrm{i})$ ). Taken together, these results suggest that the protective effects of KLF2 overexpression in $\mathrm{OA}$ are associated with the regulation of the Nrf2/ARE signaling pathway in vitro.

3.5. KLF2 Protects against MIA-Induced Cartilage Destruction in a Rat OA Model. The potential effects of KLF2 upregulation as a new therapeutic strategy were analyzed in OA animals (Figure 5(a)). We overexpressed KLF2 in knee joint cartilage tissues of 8 -week-old male rats via IA injection of an adenovirus expressing KLF2 (Ad-KLF2). Previous studies have demonstrated that an adenovirus system effectively delivers genes to knee joint cartilage tissues $[25,32]$. KLF2 overexpression significantly decreased the cartilage damage caused by MIA injection, as determined by safranin $\mathrm{O}$ staining and OARSI grade scoring (Figure 5(b)). The TUNEL staining revealed that KLF2 overexpression significantly decreased the percentage of apoptotic chondrocytes caused by MIA injection (Figure 5(c)). In addition, the results from immunohistochemical staining revealed that KLF2 protein levels were significantly decreased in the MIA group compared to those in the control group (Figures 5(d) and $5(\mathrm{e}))$. By comparing the KLF2 expression levels in the MIA + Ad-C group and the MIA+Ad-KLF2 group, we also identified the successful upregulation of KLF2 expression by IA injection of Ad-KLF2 (Figures 5(d) and 5(e)). We next examined the effects of KLF2 on the expression of MMP13 in vivo. The results showed that KLF2 overexpression significantly decreased MMP13 expression caused by MIA injection (Figures 5(d) and 5(e)). Furthermore, immunohistochemical staining revealed that KLF2 overexpression effectively promoted the nuclear translocation of Nrf2 in vivo (Figure 5(f)). To further demonstrate that the therapeutic effect of KLF2 is partly Nrf2-dependent, we inhibited Nrf2 expression using ML385 through IA injection. The results showed that Nrf2 
Treatment with MIA, adenovirus and ML385

IA injection of MIA or saline
Adaption to IA injection of ML385 two
the laboratory times a week for 4 weeks
condition

(a)
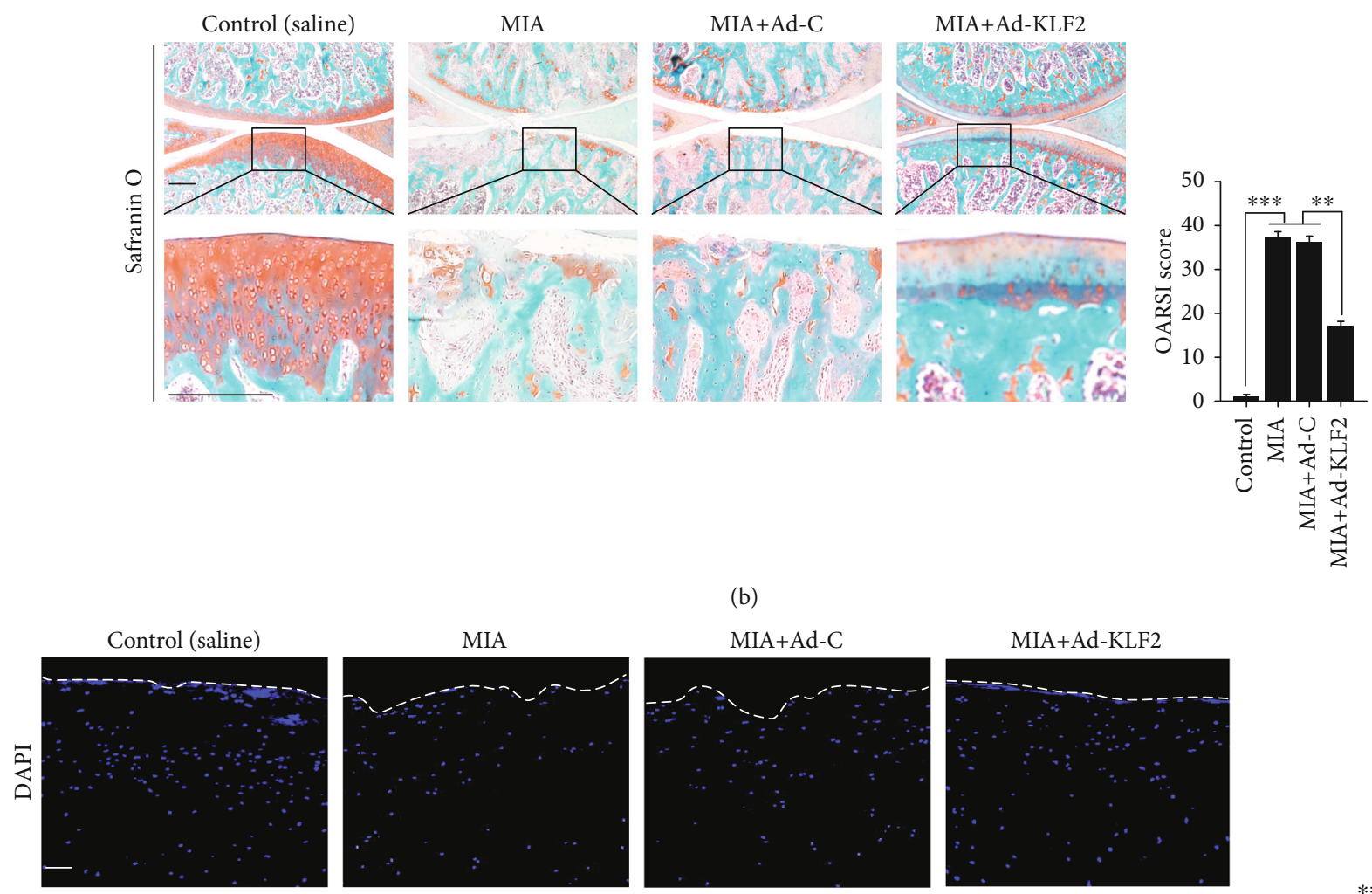

(b)
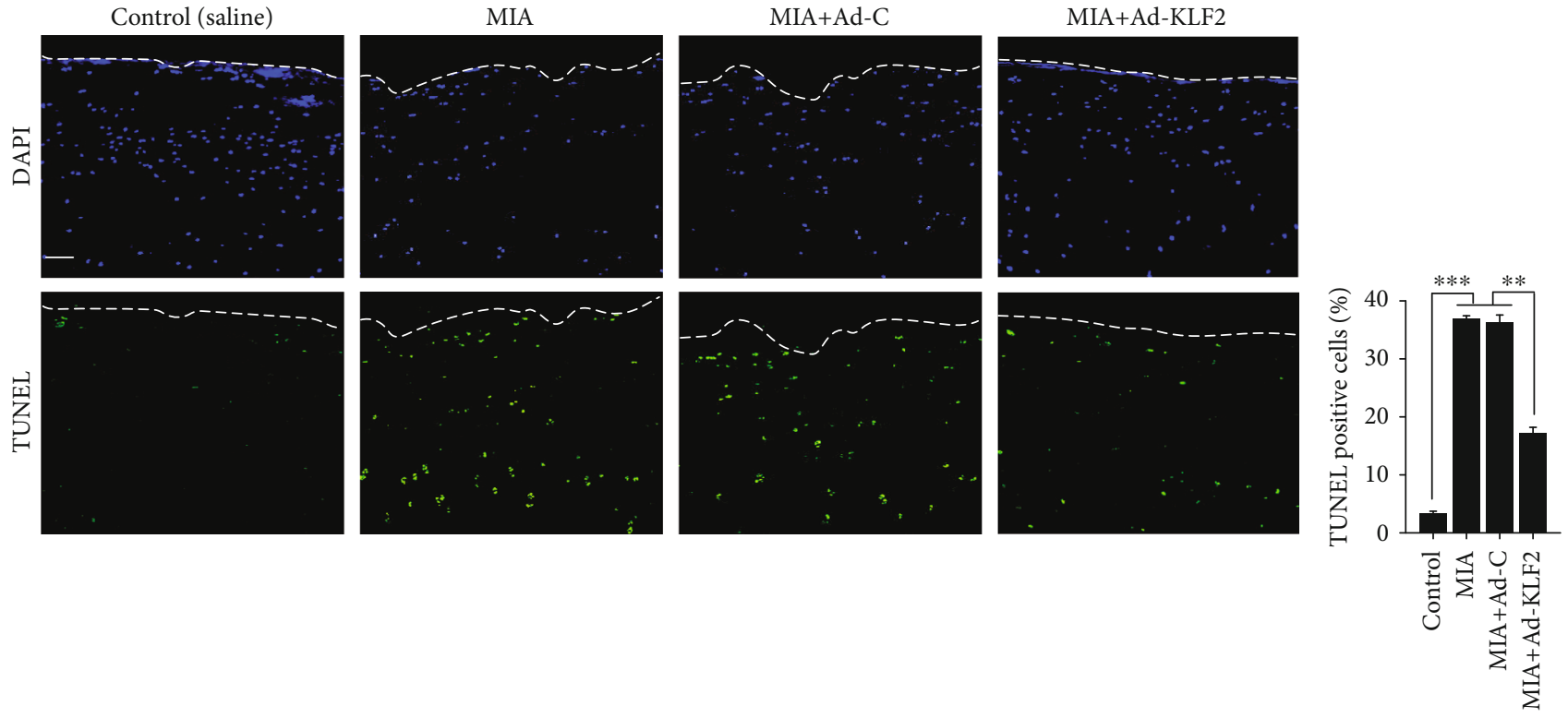

(c)

Figure 5: Continued. 


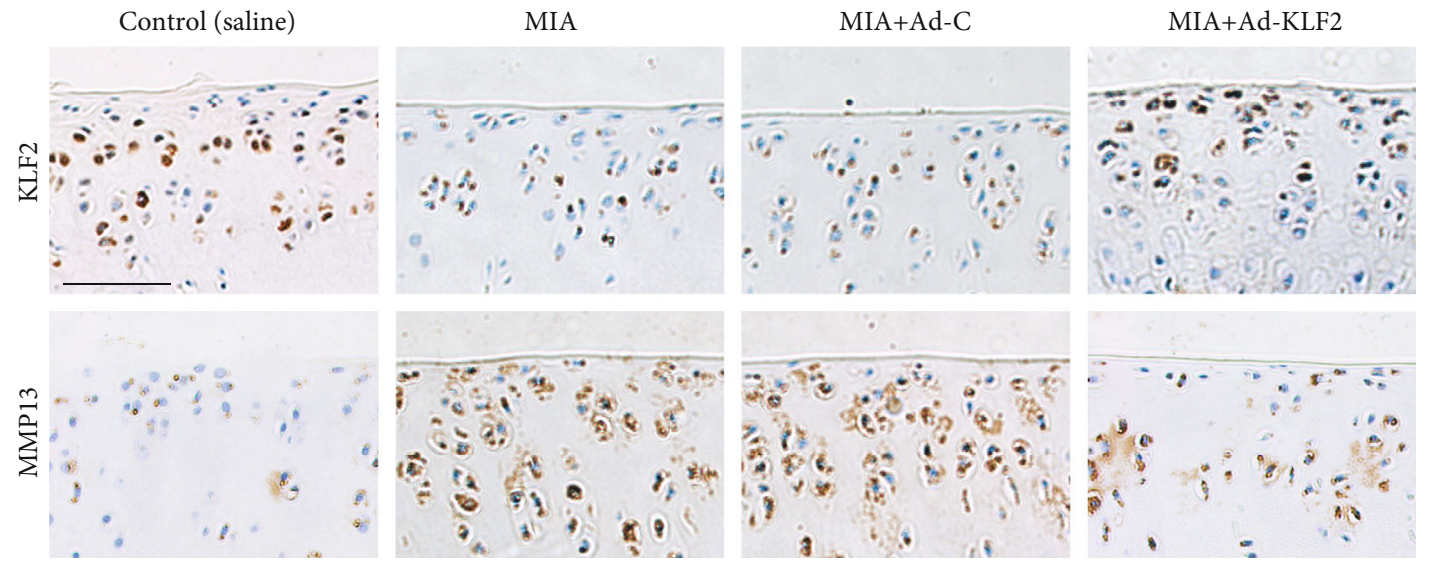

(d)

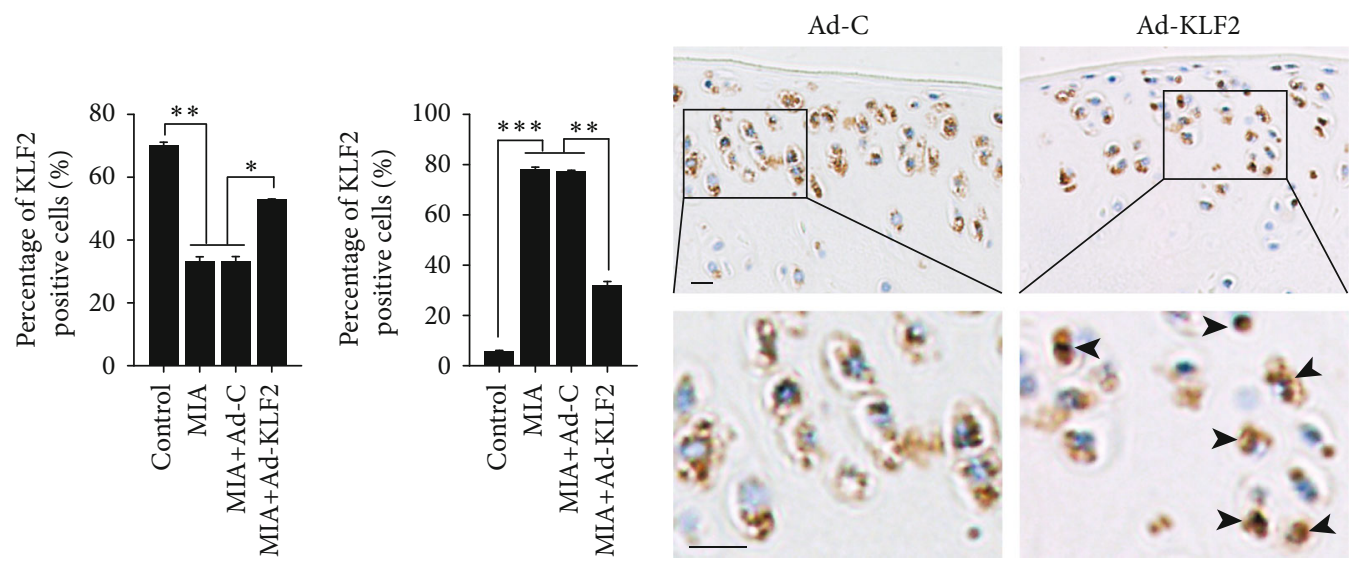

(e)

(f)
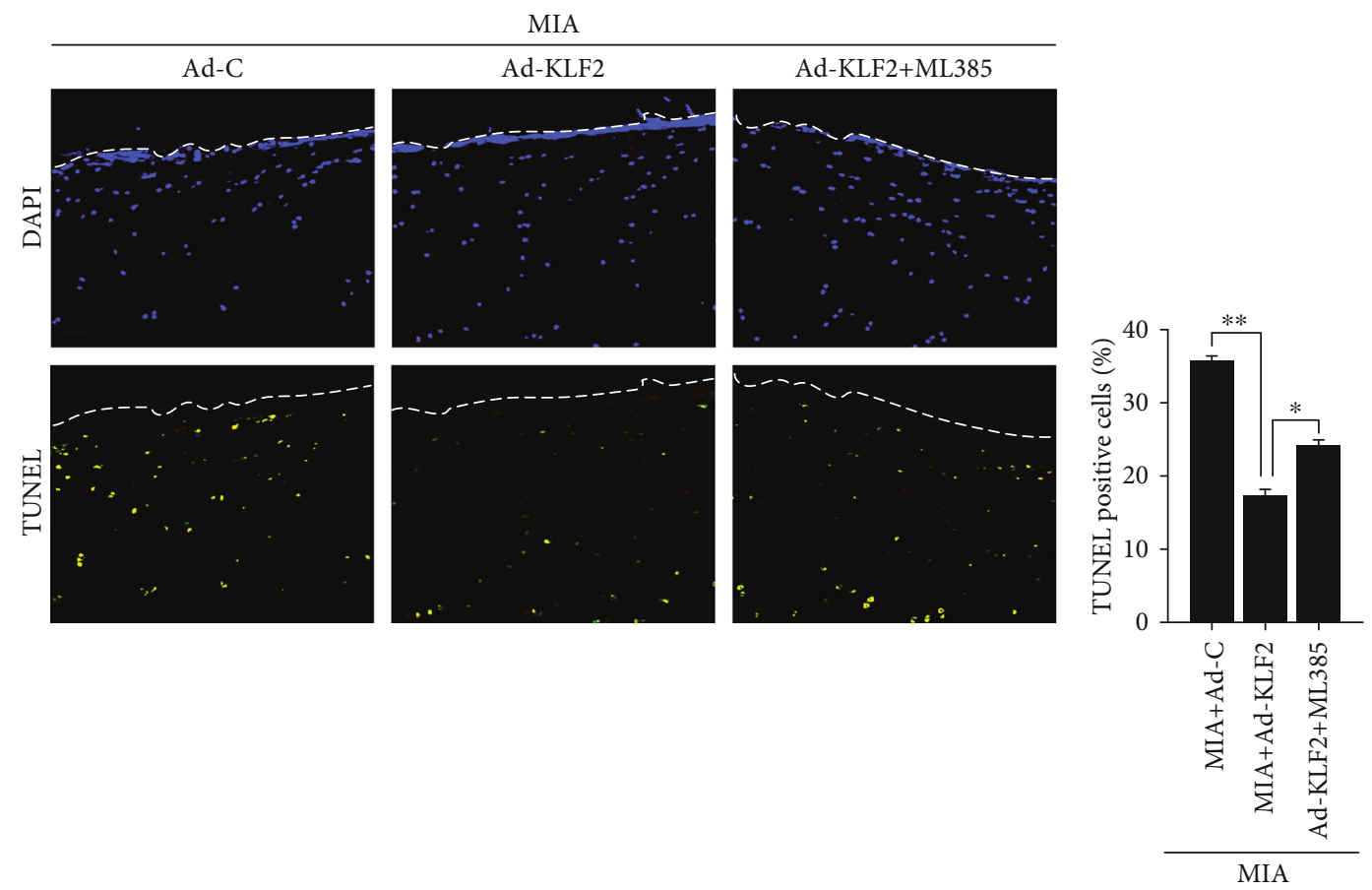

(g)

Figure 5: Continued. 


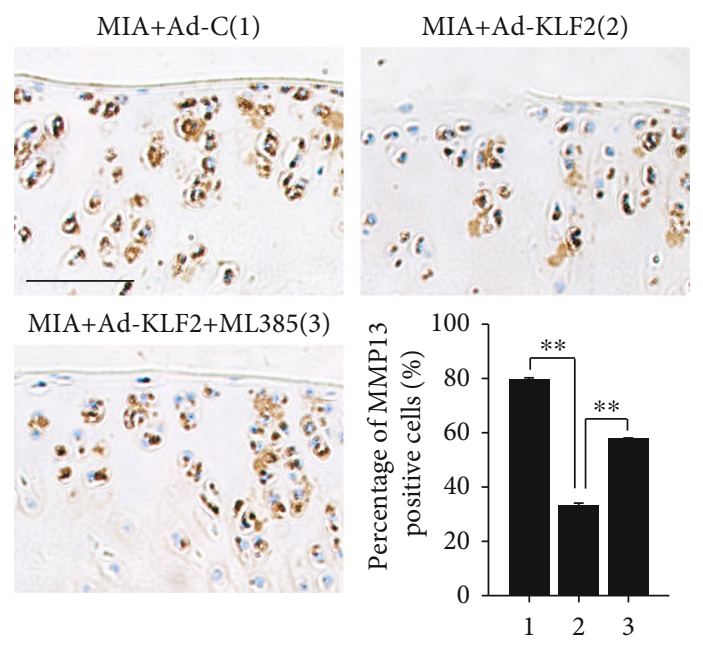

(h)

FIgURE 5: KLF2 protects against MIA-induced osteoarthritis in vivo. (a) Schematic depiction of the time of treatment. (b) Rats subjected to IA injection with sterile saline or MIA were IA-injected with Ad-C as a control or Ad-KLF2 to overexpress KLF2 in cartilage tissues ( $n=11$ per group). Cartilage destruction was determined by safranin O staining and evaluated by OARSI grade. The scale bar represents $200 \mu \mathrm{m}$. (c) Detection and quantitation of apoptotic chondrocytes in cartilage by TUNEL staining. The scale bar represents $100 \mu \mathrm{m}$. (d) Expression of KLF2 and MMP13 was evaluated by immunohistochemical staining. The scale bar represents $50 \mu \mathrm{m}$. (e) Quantification of the immunohistochemical staining of KLF2 and MMP13. (f) Expression of Nrf2 was evaluated by immunohistochemical staining. Black arrowheads indicate the cells with markedly increased expression levels of nuclear Nrf2. The scale bar represents $10 \mu \mathrm{m}$. (g) Rats subjected to IA injection with MIA were IA-injected with Ad-C as a control or Ad-KLF2 to overexpress KLF2 in cartilage tissues with or without IA injection of ML385 ( $n=11$ per group). Detection and quantitation of apoptotic chondrocytes in cartilage by TUNEL staining. The scale bar represents $100 \mu \mathrm{m}$. (h) Expression of MMP13 was evaluated by immunohistochemical staining. The scale bar represents $50 \mu \mathrm{m}$. All data are expressed as the mean \pm SD. ${ }^{*} p<0.05,{ }^{* *} p<0.005$, and ${ }^{* * *} p<0.0005$.

inhibition significantly abrogated the KLF2-mediated suppression of apoptosis and MMP13 induced by MIA in vivo (Figures 5(g) and 5(h)). These results indicate that KLF2 acts as a catabolic regulator of cartilage degeneration and $\mathrm{OA}$ pathogenesis and that KLF2 could be a potential therapeutic target for OA.

\section{Discussion}

Although several factors, such as age, genetic heritage, mechanical stress, trauma, and metabolism, are associated with OA progression, the alterations in cell signaling and metabolism that occur in chondrocytes are not yet fully known. In the present study, we uncovered that KLF2 expression is decreased during OA progression and that the modulation of KLF2 levels alters cartilage homeostasis by regulating apoptosis and ECM degradation through the regulation of ROS production. Mechanistically, we found that KLF2 overexpression promotes the nuclear translocation of Nrf2. We then further employed OA animal models to verify the potential therapeutic effect of KLF2 in OA progression.

KLFs are zinc finger family members, which serve as critical transcription factors in various biological processes [33]. Among the family members, KLF2 has been the most widely studied for its role in the activation of immune cells and the regulation of inflammation [34]. To date, two studies have reported the pathological and biological role of KLF2 in rheumatoid arthritis (RA). One study reported that the upregulation of KLF2 is associated with the downregulation of MMPs in monocytes [17]. Another study showed that KLF2 has the potential to attenuate the inflammatory properties of monocytes and regulate monocyte differentiation in the context of RA [13]. Although RA and OA share many similar features of joint damage, they represent different etiological and pathological processes. Whether KLF2 regulates cartilage homeostasis in OA pathogenesis has not been investigated in detail. The only study to date describing a role for KLF2 in chondrocytes is the in vitro study by Yuan et al., who reported that KLF2 regulates the degradation of COL2A1 by suppressing the IL-1 $\beta$-induced expression of MMP13 [20]. A relatively large number of studies have reported that KLF2 expression is consistently reduced across a diverse array of acute or chronic inflammatory conditions [13, 16, 17, 35]. Consistent with these previous reports, our initial analysis using microarray (GSE114007) and clinical cartilage samples revealed that KLF2 is downregulated in human OA cartilage. In addition, our data suggest for the first time that KLF2 expression levels are significantly correlated with OA severity.

Considering this correlation between KLF2 expression and $\mathrm{OA}$ conditions, we next evaluated the possible protective effects of KLF2 in vitro under IL- $1 \beta$ stimulation conditions. IL- $1 \beta$ is a critical proinflammatory cytokine in the pathogenesis of OA and is usually utilized to stimulate chondrocytes to mimic the OA conditions in in vitro studies [11, 20,29]. In the present study, KLF2 expression was downregulated in cells stimulated with IL-1 $\beta$ in a dose- and time-dependent manner. Genetic or pharmacological overexpression of KLF2 in chondrocytes stimulated with IL- $1 \beta$ inhibited the 
expression of MMPs, including MMP3, MMP9, and MMP13, and increased the production of COL2A1. Pharmacological inhibition of KLF2 using GGPP resulted in a significant blockade of the KLF2 overexpression-induced effects on MMPs and COL2A1. These observations indicate that KLF2 functions as a critical regulator of MMPs and COL2A1, which are both crucial molecules in the OA-related degradation of the ECM $[6,7]$. To the best of our knowledge, chondrocyte apoptosis is an underlying factor for the initiation of OA $[30,36]$. Considering the antiapoptotic properties of KLF2 [37, 38], we performed flow cytometry experiments to detect changes in apoptosis. The results indicated that genetic or pharmacological overexpression of KLF2 in chondrocytes stimulated with IL- $1 \beta$ effectively reduced the rate of apoptosis. Pharmacological inhibition of KLF2 using GGPP resulted in a significant blockade of the KLF2 overexpression-induced effects on apoptosis. Emerging evidence suggests that oxidative stress-derived production of excessive ROS plays a critical role in the progression of OA by inducing chondrocyte apoptosis and ECM degradation $[5,39]$. Therefore, we hypothesized that KLF2 might exert a chondroprotective effect by regulating ROS generation. Our results, for the first time, indicate that KLF2 overexpression significantly attenuates IL- $1 \beta$-induced ROS generation in chondrocytes. In summary, these results suggest that KLF2 might be a potential therapeutic target for OA pathogenesis.

Increasing evidence supports the hypothesis that Nrf2 plays a key role in the oxidative stress response during $\mathrm{OA}$ progression and activation of Nrf2/ARE signaling is the primary method to reduce the level of $\operatorname{ROS}[9,11]$. Usually, Nrf2 is retained in the cytoplasm by binding to the cytoskeleton-associated protein Keap1 and is degraded by the proteasome. Upon stimulation, Nrf2 can be released from the Nrf2-Keap1 dimer and translocate to the nucleus where it binds to the AREs present in the promoter regions of target genes, such as HO-1 and NQO1. However, this increase in nuclear translocation of Nrf2 is not enough to offset the oxidative stress induced by OA pathogenesis [11]. Based on this evidence, we hypothesized that KLF2 might exert ECM anticatabolism and antiapoptosis effects by regulating Nrf2 activity. The results of this study indicate that overexpression of KLF2 induces the expression of downstream Nrf2 genes, including $\mathrm{HO}-1$ and NQO1, a phenomenon that was reversed by ML385 (an inhibitor of Nrf2) treatment. Western blot and immunofluorescence analysis revealed that KLF2 overexpression promotes the nuclear translocation of Nrf2, which could explain the KLF2 overexpression-induced increase in HO-1 and NQO1 expression. These results are consistent with those of previous studies showing that KLF2 exerts an antioxidative stress effect by promoting the nuclear translocation of Nrf2 $[18,19,23]$. The translocation of NRF2 is primarily regulated at the level of protein stability in the cytoplasm. Due to continuous ubiquitination and proteasomal degradation, the levels of NRF2 are low at homeostatic status. The major regulator of NRF2 degradation is Kelch-like ECH-associated protein 1 (KEAP1). When KEAP1 binds to the ETGE and the DLG motifs of NRF2, NRF2 can be ubiquitinated by cullin-3- (CUL3-) RBX1/ROC1 ubiquitin ligase and degraded by the $26 \mathrm{~S}$ pro- teasome, thus keeping Nrf2 at low levels in the cytoplasm [40]. Another way of NRF2 degradation is mediated by glycogen synthase kinase 3- (GSK-3-) dependent phosphorylation [41]. The degradation of NRF2 also can be regulated by acetylation through histone acetyl transferases p300 [42]. However, at the present study, the underlying mechanism between KLF2 and Nrf2 remains unknown, which should be further explored in the future. One recent study reported that overexpression of KLF2 decreased the levels of H4K8 and $\mathrm{H} 3 \mathrm{~K} 9$ acetylation [43]. Wu et al. demonstrated that the phosphorylation activity of p38 could be regulated by KLF2 [44]. Furthermore, a previous study highlighted that KLF2 can promote HIF-1 $\alpha$ degradation through a proteasomedependent manner [45]. Therefore, these approaches might be the orientations to fully understand the role of KLF2 and Nrf2/ARE signaling in OA. Further research will be required to determine the regulatory mechanism between KLF2 and Nrf2 as a therapeutic target.

To further ascertain that Nrf2 is involved in the chondroprotective effect of KLF2, we inhibited Nrf2 expression using ML385. As expected, the inhibition of MMP13 and apoptosis triggered by KLF2 overexpression was abolished by the inhibition of Nrf2. Taken together, these results demonstrate for the first time that overexpression of KLF2 in chondrocytes results in the inhibition of apoptosis and ECM degradation through Nrf2 activation.

Previously, studies have reported that simvastatin significantly inhibits the IL- $1 \beta$-induced production of matrixdegrading enzymes in vitro and protects against the development of cartilage degeneration in OA model mice $[46,47]$. It has also been reported that simvastatin triggers the nuclear translocation of Nrf2 in the rat liver [48]. However, the mechanism of simvastatin-mediated protection against the development of OA has not been elucidated in previous studies. In the present study, simvastatin was used as a pharmacological inducer of KLF2 for in vitro experiments, and the interesting findings herein are consistent with the findings in these previous reports. Thus, our data indicate that the chondroprotective effect of simvastatin reported in previous studies may be mediated by KLF2.

To further investigate the chondroprotective effect of KLF2, we used an OA animal model to assess the potential therapeutic effects of KLF2 on OA pathogenesis. We induced OA by IA injection of MIA, which has been widely used to investigate OA pathogenesis $[49,50]$. Previous studies have extensively demonstrated that adenoviruses can effectively deliver target genes to cartilage tissue [25, 51, 52]. For instance, Son et al. reported that three weekly IA injections of Ad-HSPA1A triggered the effective overexpression of HSPA1A in the cartilage, which clearly abrogated the cartilage erosion caused by OA progression [25]. In the present study, KLF2 overexpression in joint tissues effectively abrogated cartilage degradation caused by IA injection of MIA. Immunohistochemical staining indicated that KLF2 protein levels were significantly decreased in the OA rat tissue. In addition, the adenovirus-mediated overexpression of KLF2 in rat knee joint cartilage downregulated the expression of MMP13. Importantly, we further confirmed that KLF2 overexpression in joint cartilage tissues could effectively promote 
Nrf2 translocation in vivo and Nrf2 inhibition significantly abrogated the KLF2-mediated suppression of apoptosis and MMP13 induced by MIA in vivo.

This in vitro and in vivo study is the first to investigate the chondroprotective effect and mechanisms of KLF2 in OA pathogenesis. However, there were several limitations to this study. On the one hand, the specific regulatory mechanism between KLF2 and Nrf2 remains to be explored, which is the focus of our next study. However, we did not address the involvement of Keap1, which has been identified as an indispensable mediator of the Nrf2-Keap1 redox signaling pathway.

\section{Conclusions}

In summary, our study shows for the first time that KLF2 expression is decreased in OA and closely associated with the severity of OA. KLF2 plays a pivotal role in protecting chondrocytes against IL- $1 \beta$-induced apoptosis and ECM degradation by suppressing ROS generation in vitro. Mechanistically, we show that KLF2 overexpression effectively activates Nrf2/ARE signaling to promote the transcription of Nrf2 target genes, hence repressing oxidative stress. Additionally, the overexpression of KLF2 in joint tissues is sufficient to alleviate experimental $\mathrm{OA}$ in rats. Our results collectively provide new insights into OA pathogenesis regulated by KLF2 and a rationale for the development of effective intervention strategies for OA.

\section{Data Availability}

The data used to support the findings of this study are available from the corresponding author upon request.

\section{Conflicts of Interest}

The authors declare that there is no conflict of interest regarding the publication of this paper.

\section{Acknowledgments}

This study is funded by the Natural Science Foundation of Liaoning Province (CN) (2015020473) and National Natural Science Foundation of China (81272946). We thank Shang Piao for his help with the rat work.

\section{Supplementary Materials}

Figure S1: coimmunoprecipitation measuring the interaction of KLF2 and Nrf2. (Supplementary Materials)

\section{References}

[1] E. Thomas, G. Peat, and P. Croft, "Defining and mapping the person with osteoarthritis for population studies and public health," Rheumatology, vol. 53, no. 2, pp. 338-345, 2014.

[2] M. Husa, R. Liu-Bryan, and R. Terkeltaub, "Shifting HIFs in osteoarthritis," Nature Medicine, vol. 16, no. 6, pp. 641-644, 2010.
[3] A. C. Lin, B. L. Seeto, J. M. Bartoszko et al., "Modulating hedgehog signaling can attenuate the severity of osteoarthritis," Nature Medicine, vol. 15, no. 12, pp. 1421-1425, 2009.

[4] L. Troeberg and H. Nagase, "Proteases involved in cartilage matrix degradation in osteoarthritis," Biochimica et Biophysica Acta (BBA) - Proteins and Proteomics, vol. 1824, no. 1, pp. 133-145, 2012.

[5] A. S. Marchev, P. A. Dimitrova, A. J. Burns, R. V. Kostov, A. T. Dinkova-Kostova, and M. I. Georgiev, "Oxidative stress and chronic inflammation in osteoarthritis: can NRF2 counteract these partners in crime?," Annals of the New York Academy of Sciences, vol. 1401, no. 1, pp. 114-135, 2017.

[6] A. B. Blom, P. L. van Lent, S. Libregts et al., "Crucial role of macrophages in matrix metalloproteinase-mediated cartilage destruction during experimental osteoarthritis : Involvement of matrix metalloproteinase 3," Arthritis and Rheumatism, vol. 56, no. 1, pp. 147-157, 2007.

[7] C. B. Little, A. Barai, D. Burkhardt et al., "Matrix metalloproteinase 13-deficient mice are resistant to osteoarthritic cartilage erosion but not chondrocyte hypertrophy or osteophyte development," Arthritis and Rheumatism, vol. 60, no. 12, pp. 3723-3733, 2009.

[8] E. Charlier, B. Relic, C. Deroyer et al., "Insights on molecular mechanisms of chondrocytes death in osteoarthritis," International Journal of Molecular Sciences, vol. 17, no. 12, p. 2146, 2016.

[9] P. Lepetsos and A. G. Papavassiliou, "ROS/oxidative stress signaling in osteoarthritis," Biochimica et Biophysica Acta (BBA) Molecular Basis of Disease, vol. 1862, no. 4, pp. 576-591, 2016.

[10] J. A. Collins, B. O. Diekman, and R. F. Loeser, "Targeting aging for disease modification in osteoarthritis," Current Opinion in Rheumatology, vol. 30, no. 1, pp. 101-107, 2018.

[11] N. M. Khan, I. Ahmad, and T. M. Haqqi, "Nrf2/ARE pathway attenuates oxidative and apoptotic response in human osteoarthritis chondrocytes by activating ERK1/2/ELK1-P70S6KP90RSK signaling axis," Free Radical Biology \& Medicine, vol. 116, pp. 159-171, 2018.

[12] D. Cai, S. Yin, J. Yang, Q. Jiang, and W. Cao, "Histone deacetylase inhibition activates Nrf2 and protects against osteoarthritis," Arthritis Research \& Therapy, vol. 17, no. 1, p. 269, 2015.

[13] M. Das, J. Lu, M. Joseph et al., "Kruppel-like factor 2 (KLF2) regulates monocyte differentiation and functions in $\mathrm{mBSA}$ and IL- $1 \beta$-induced arthritis," Current Molecular Medicine, vol. 12, no. 2, pp. 113-125, 2012.

[14] P. Novodvorsky and T. J. Chico, "The Role of the Transcription Factor KLF2 in Vascular Development and Disease," Progress in Molecular Biology and Translational Science, vol. 124, pp. 155-188, 2014.

[15] F. Zhong, H. Chen, C. Wei et al., "Reduced Krüppel-like factor 2 expression may aggravate the endothelial injury of diabetic nephropathy," Kidney International, vol. 87, no. 2, pp. 382$395,2015$.

[16] H. Das, A. Kumar, Z. Lin et al., "Kruppel-like factor 2 (KLF2) regulates proinflammatory activation of monocytes," Proceedings of the National Academy of Sciences, vol. 103, no. 17, pp. 6653-6658, 2006.

[17] M. Das, D. Laha, S. Kanji et al., "Induction of Krüppel-like factor 2 reduces $\mathrm{K} / \mathrm{BxN}$ serum-induced arthritis," Journal of Cellular and Molecular Medicine, vol. 23, no. 2, pp. 13861395, 2019. 
[18] J. O. Fledderus, R. A. Boon, O. L. Volger et al., "KLF2 primes the antioxidant transcription factor Nrf2 for activation in endothelial cells," Arteriosclerosis, Thrombosis, and Vascular Biology, vol. 28, no. 7, pp. 1339-1346, 2008.

[19] W. Wu, P. Geng, J. Zhu et al., "KLF2 regulates eNOS uncoupling via Nrf2/HO-1 in endothelial cells under hypoxia and reoxygenation," Chemico-Biological Interactions, vol. 305, pp. 105-111, 2019.

[20] Y. Yuan, H. Tan, and P. Dai, "Krüppel-Like factor 2 regulates degradation of type II collagen by suppressing the expression of matrix metalloproteinase (MMP)-13," Cellular Physiology and Biochemistry, vol. 42, no. 6, pp. 2159-2168, 2017.

[21] H. J. Mankin, "Biochemical and metabolic abnormalities in osteoarthritic human cartilage," Federation Proceedings, vol. 32, no. 4, pp. 1478-1480, 1973.

[22] W. S. Lian, J. Y. Ko, R. W. Wu et al., "MicroRNA-128a represses chondrocyte autophagy and exacerbates knee osteoarthritis by disrupting Atg12," Cell Death \& Disease, vol. 9, no. 9, p. 919, 2018.

[23] G. Marrone, R. Maeso-Díaz, G. García-Cardena et al., "KLF2 exerts antifibrotic and vasoprotective effects in cirrhotic rat livers: behind the molecular mechanisms of statins," Gut, vol. 64, no. 9, pp. 1434-1443, 2015.

[24] T. T. Tuomisto, H. Lumivuori, E. Kansanen et al., "Simvastatin has an anti-inflammatory effect on macrophages via upregulation of an atheroprotective transcription factor, Kruppel-like factor 2," Cardiovascular Research, vol. 78, no. 1, pp. 175$184,2008$.

[25] Y. O. Son, H. E. Kim, W. S. Choi, C. H. Chun, and J. S. Chun, "RNA-binding protein ZFP36L1 regulates osteoarthritis by modulating members of the heat shock protein 70 family," Nature Communications, vol. 10, no. 1, p. 77, 2019.

[26] N. Gerwin, A. M. Bendele, S. Glasson, and C. S. Carlson, “The OARSI histopathology initiative - recommendations for histological assessments of osteoarthritis in the rat," Osteoarthritis and Cartilage, vol. 18, Suppl 3, pp. S24-S34, 2010.

[27] N. Holzer, D. Salvo, A. C. Marijnissen et al., "Radiographic evaluation of posttraumatic osteoarthritis of the ankle: the Kellgren-Lawrence scale is reliable and correlates with clinical symptoms," Osteoarthritis and Cartilage, vol. 23, no. 3, pp. 363-369, 2015.

[28] C. A. Dinarello, "Immunological and inflammatory functions of the interleukin-1 family," Annual Review of Immunology, vol. 27, no. 1, pp. 519-550, 2009.

[29] Y. Jiang, C. Hu, S. Yu et al., "Cartilage stem/progenitor cells are activated in osteoarthritis via interleukin- $1 \beta /$ nerve growth factor signaling," Arthritis Research \& Therapy, vol. 17, no. 1, p. $327,2015$.

[30] C. M. Thomas, C. J. Fuller, C. E. Whittles, and M. Sharif, "Chondrocyte death by apoptosis is associated with cartilage matrix degradation," Osteoarthritis and Cartilage, vol. 15, no. 1, pp. 27-34, 2007.

[31] S. Guixe-Muntet, F. C. de Mesquita, S. Vila et al., "Crosstalk between autophagy and KLF2 determines endothelial cell phenotype and microvascular function in acute liver injury," Journal of Hepatology, vol. 66, no. 1, pp. 86-94, 2017.

[32] Y. O. Son, S. Park, J. S. Kwak et al., "Estrogen-related receptor $\gamma$ causes osteoarthritis by upregulating extracellular matrixdegrading enzymes," Nature Communications, vol. 8, no. 1, p. $2133,2017$.
[33] B. B. McConnell and V. W. Yang, "Mammalian Krüppel-like factors in health and diseases," Physiological Reviews, vol. 90, no. 4, pp. 1337-1381, 2010.

[34] P. Jha and H. Das, "KLF2 in regulation of NF- $\kappa$ B-Mediated immune cell function and inflammation," International Journal of Molecular Sciences, vol. 18, no. 11, p. 2383, 2017.

[35] G. H. Mahabeleshwar, D. Kawanami, N. Sharma et al., "The myeloid transcription factor KLF2 regulates the host response to polymicrobial infection and endotoxic shock," Immunity, vol. 34, no. 5, pp. 715-728, 2011.

[36] H. S. Hwang and H. A. Kim, "Chondrocyte apoptosis in the pathogenesis of osteoarthritis," International Journal of Molecular Sciences, vol. 16, no. 11, pp. 26035-26054, 2015.

[37] Y. Lin, J. Ryan, J. Lewis, M. A. Wani, J. B. Lingrel, and Z. G. Liu, "TRAF2 exerts its antiapoptotic effect by regulating the expression of Kruppel-like factor LKLF," Molecular and Cellular Biology, vol. 23, no. 16, pp. 5849-5856, 2003.

[38] Z. Liu, X. Zhang, Q. Xiao et al., "Pretreatment donors after circulatory death with simvastatin alleviates liver ischemia reperfusion injury through a KLF2-dependent mechanism in rat," Oxidative Medicine and Cellular Longevity, vol. 2017, Article ID 3861914, 10 pages, 2017.

[39] M. Del Carlo Jr. and R. F. Loeser, "Nitric oxide-mediated chondrocyte cell death requires the generation of additional reactive oxygen species," Arthritis and Rheumatism, vol. 46, no. 2, pp. 394-403, 2002.

[40] M. McMahon, N. Thomas, K. Itoh, M. Yamamoto, and J. D. Hayes, "Dimerization of substrate adaptors can facilitate cullin-mediated ubiquitylation of proteins by a "tethering" mechanism: a two-site interaction model for the Nrf2-Keap1 complex," The Journal of Biological Chemistry, vol. 281, no. 34, pp. 24756-24768, 2006.

[41] P. Rada, A. I. Rojo, S. Chowdhry, M. McMahon, J. D. Hayes, and A. Cuadrado, "SCF/ $\beta$-TrCP promotes glycogen synthase kinase 3-dependent degradation of the Nrf2 transcription factor in a Keap1-independent manner," Molecular and Cellular Biology, vol. 31, no. 6, pp. 1121-1133, 2011.

[42] Z. Sun, Y. E. Chin, and D. D. Zhang, "Acetylation of Nrf2 by p300/CBP augments promoter-specific DNA binding of Nrf2 during the antioxidant response," Molecular and Cellular Biology, vol. 29, no. 10, pp. 2658-2672, 2009.

[43] D. Laha, M. Deb, and H. Das, "KLF2 (Kruppel like factor 2 [lung]) regulates osteoclastogenesis by modulating autophagy," Autophagy, vol. 1-13, 2019.

[44] X. Wu, J. Z. Zhang, P. F. Yang, Q. H. Huang, and J. M. Liu, "Regulation of Kruppel-like factor 2 (KLF2) in the pathogenesis of intracranial aneurysm induced by hemodynamics," American Journal of Translational Research, vol. 9, no. 12, pp. 5452-5460, 2017.

[45] D. Kawanami, G. H. Mahabeleshwar, Z. Lin et al., "Kruppellike factor 2 inhibits hypoxia-inducible factor lalpha expression and function in the endothelium," The Journal of Biological Chemistry, vol. 284, no. 31, pp. 20522-20530, 2009.

[46] T. Tanaka, T. Matsushita, K. Nishida et al., "Attenuation of osteoarthritis progression in mice following intra-articular administration of simvastatin-conjugated gelatin hydrogel," Journal of Tissue Engineering and Regenerative Medicine, vol. 13, no. 3, pp. 423-432, 2019.

[47] K. Yudoh and R. Karasawa, "Statin prevents chondrocyte aging and degeneration of articular cartilage in osteoarthritis (OA)," Aging, vol. 2, no. 12, pp. 990-998, 2010. 
[48] I. G. Habeos, P. G. Ziros, D. Chartoumpekis, A. Psyrogiannis, V. Kyriazopoulou, and A. G. Papavassiliou, "Simvastatin activates Keap1/Nrf2 signaling in rat liver," Journal of Molecular Medicine, vol. 86, no. 11, pp. 1279-1285, 2008.

[49] S. Ashraf, P. I. Mapp, J. Burston, A. J. Bennett, V. Chapman, and D. A. Walsh, "Augmented pain behavioural responses to intra-articular injection of nerve growth factor in two animal models of osteoarthritis," Annals of the Rheumatic Diseases, vol. 73, no. 9, pp. 1710-1718, 2014.

[50] D. R. Sagar, S. Ashraf, L. Xu et al., "Osteoprotegerin reduces the development of pain behaviour and joint pathology in a model of osteoarthritis," Annals of the Rheumatic Diseases, vol. 73, no. 8, pp. 1558-1565, 2014.

[51] J. H. Kim, J. Jeon, M. Shin et al., "Regulation of the catabolic cascade in osteoarthritis by the zinc-ZIP8-MTF1 axis," Cell, vol. 156, no. 4, pp. 730-743, 2014.

[52] Y. Won, Y. Shin, C. H. Chun et al., "Pleiotropic roles of metallothioneins as regulators of chondrocyte apoptosis and catabolic and anabolic pathways during osteoarthritis pathogenesis," Annals of the Rheumatic Diseases, vol. 75, no. 11, pp. 2045-2052, 2016. 


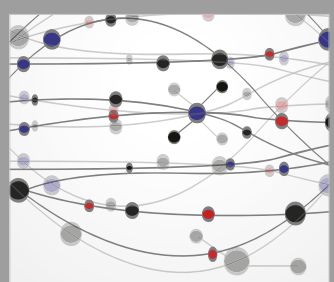

The Scientific World Journal
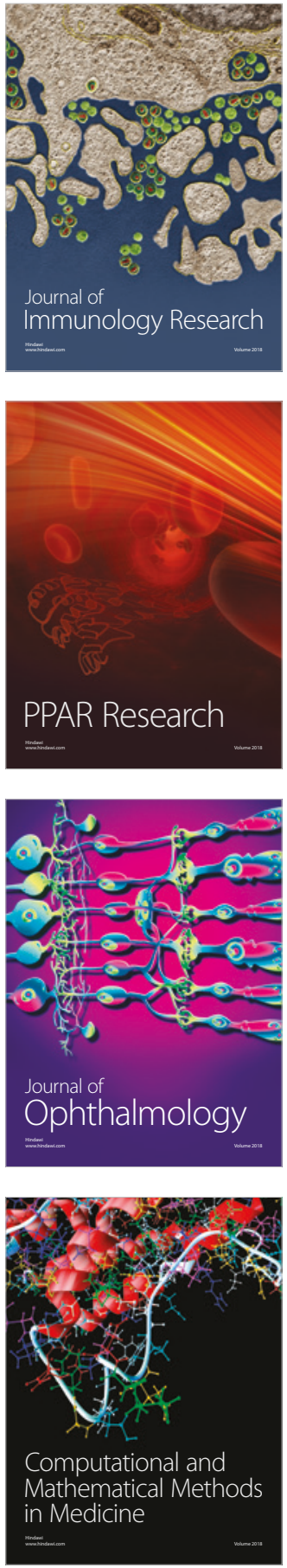

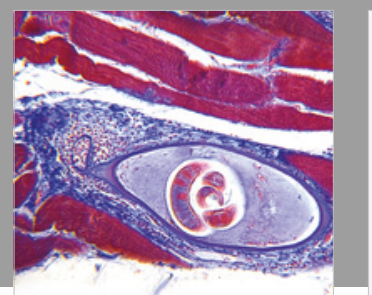

Gastroenterology Research and Practice

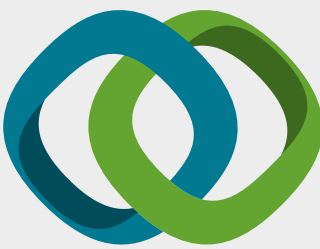

\section{Hindawi}

Submit your manuscripts at

www.hindawi.com
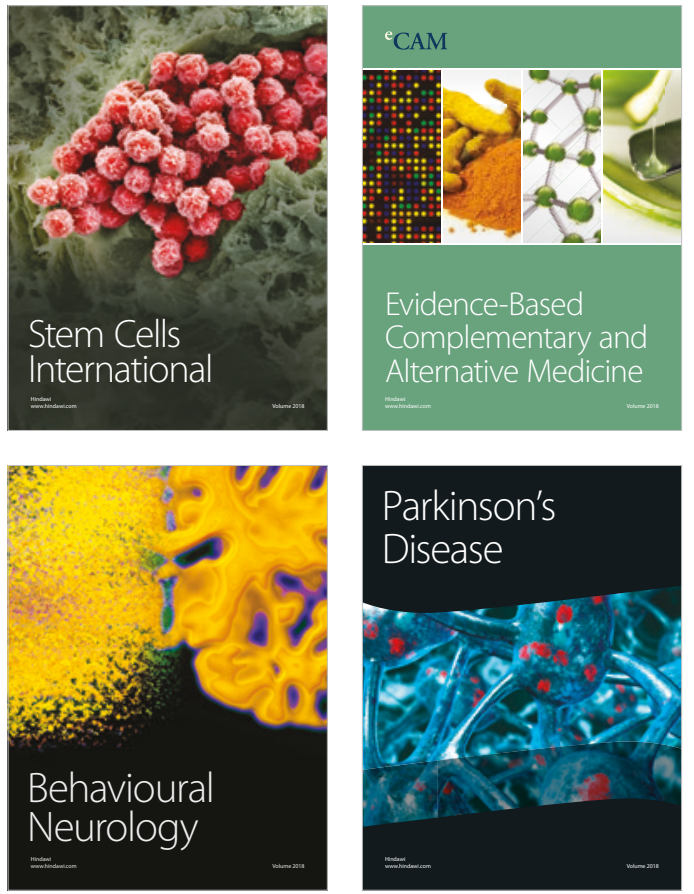

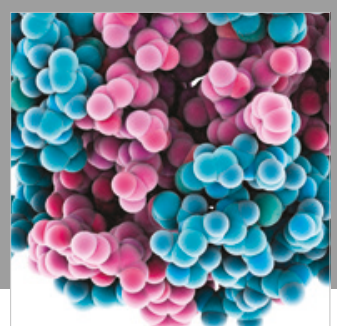

ournal of

Diabetes Research

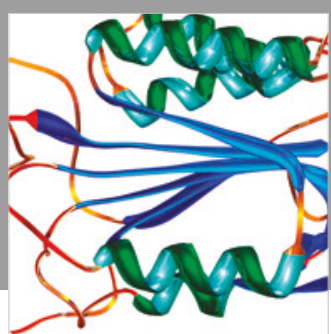

Disease Markers
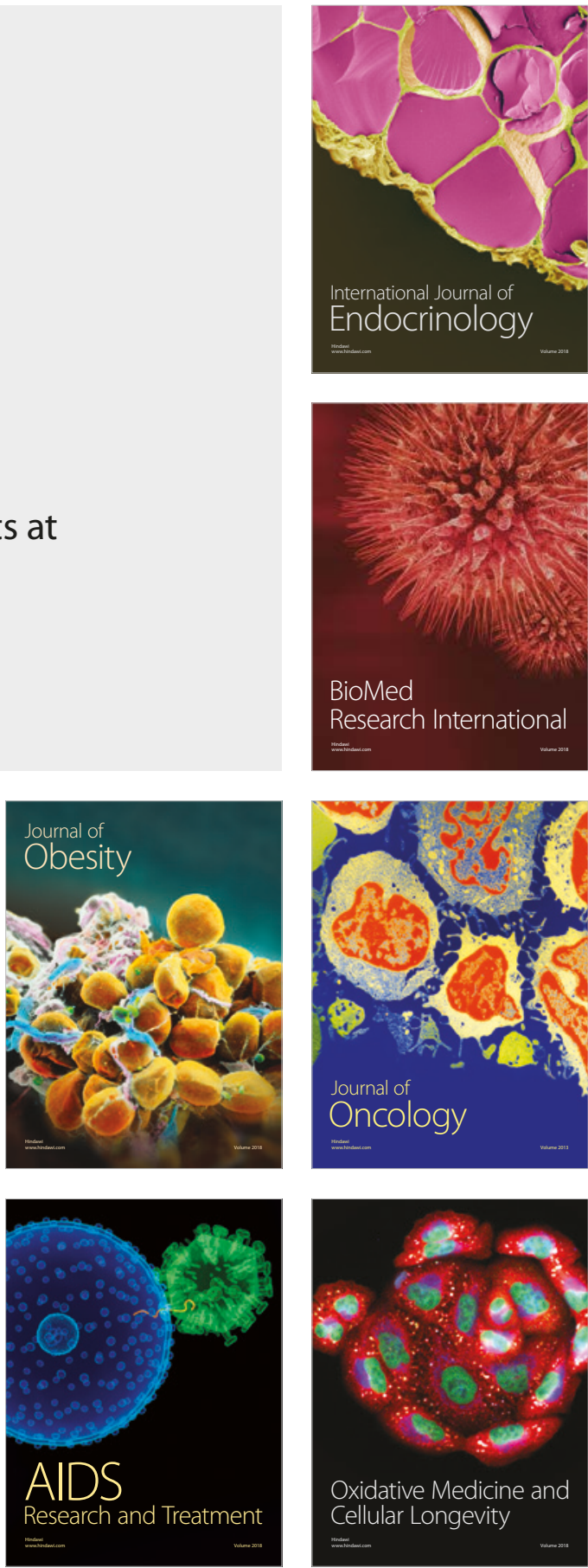